\title{
Classification of a Subclass of Two-Dimensional Lattices via Characteristic Lie Rings
}

\author{
Ismagil HABIBULLIN ${ }^{\dagger}$ and Mariya POPTSOVA ${ }^{\dagger}$ \\ † Ufa Institute of Mathematics, 112 Chernyshevsky Str., Ufa 450008, Russia \\ E-mail: habibullinismagil@gmail.com \\ E-mail: mnpoptsova@gmail.com \\ ¥ Bashkir State University, 32 Validy Str., Ufa 450076, Russia
}

Received March 30, 2017, in final form August 24, 2017; Published online September 07, 2017

https://doi.org/10.3842/SIGMA.2017.073

\begin{abstract}
The main goal of the article is testing a new classification algorithm. To this end we apply it to a relevant problem of describing the integrable cases of a subclass of twodimensional lattices. By imposing the cut-off conditions $u_{-1}=c_{0}$ and $u_{N+1}=c_{1}$ we reduce the lattice $u_{n, x y}=\alpha\left(u_{n+1}, u_{n}, u_{n-1}\right) u_{n, x} u_{n, y}$ to a finite system of hyperbolic type PDE. Assuming that for each natural $N$ the obtained system is integrable in the sense of Darboux we look for $\alpha$. To detect the Darboux integrability of the hyperbolic type system we use an algebraic criterion of Darboux integrability which claims that the characteristic Lie rings of such a system must be of finite dimension. We prove that up to the point transformations only one lattice in the studied class passes the test. The lattice coincides with the earlier found Ferapontov-Shabat-Yamilov equation. The one-dimensional reduction $x=y$ of this lattice passes also the symmetry integrability test.
\end{abstract}

Key words: two-dimensional integrable lattice; cut-off boundary condition; open chain; Darboux integrable system; characteristic Lie ring

2010 Mathematics Subject Classification: 37K10; 37K30; 37D99

\section{Introduction}

In the present article we study the classification problem for the following class of two-dimensional lattices

$$
u_{n, x y}=\alpha\left(u_{n+1}, u_{n}, u_{n-1}\right) u_{n, x} u_{n, y} .
$$

Here the sought function $u=u_{n}(x, y)$ depends on real $x, y$ and on integer $n$. Function $\alpha=$ $\alpha\left(u_{n+1}, u_{n}, u_{n-1}\right)$ is assumed to be analytical in a domain $D \subset \mathbb{C}^{3}$. We request also that the derivatives $\frac{\partial \alpha\left(u_{n+1}, u_{n}, u_{n-1}\right)}{\partial u_{n+1}}$ and $\frac{\partial \alpha\left(u_{n+1}, u_{n}, u_{n-1}\right)}{\partial u_{n-1}}$ do not vanish identically.

Constraint $u_{n_{0}}=c_{0}$ where $c_{0}$ is a constant parameter defines a boundary condition which cuts off the lattice (1.1) into two independent semi-infinite lattices

$$
\begin{aligned}
& u_{n, x y}=\alpha\left(u_{n+1}, u_{n}, u_{n-1}\right) u_{n, x} u_{n, y}, \quad \text { for } \quad n>n_{0} \quad\left(n<n_{0}\right), \\
& u_{n_{0}}=c_{0} .
\end{aligned}
$$

Any solutions of the lattice located on the semiaxis $n>n_{0}$ does not depend on the solutions of that located on $n<n_{0}$ and vice versa. Turning to the general case of the lattices recall that the boundary conditions (or cut-off constraints) having such a property are called degenerate. It is well known that the degenerate boundary conditions are admitted by any integrable nonlinear

This paper is a contribution to the Special Issue on Symmetries and Integrability of Difference Equations. The full collection is available at http://www.emis.de/journals/SIGMA/SIDE12.html 
lattice. They are compatible with the whole hierarchy of the higher symmetries $[1,8]$. In the literature they are met in the connection with the so-called open chains (see, for instance, [15]). Since the symmetry approach which is a powerful classification tool in the dimension $1+1$ (see, for instance, $[2,11,13]$ ) loses its efficiency in higher dimensions (an explanation can be found in [14]) it became clear years ago that it is necessary to look for alternative classification algorithms. Since then different approaches to the integrable multidimensional models have been invented (see, for instance, [3, 5, 6, 12, 16, 17, 18, 24]).

In 1994 A.B. Shabat posed a problem of creating a classification algorithm by combining the concepts of the degenerate boundary condition, open chain and the characteristic Lie algebra. It is worth mentioning as an important step in this direction the article [19] where the structure of the Lie algebra was described for the two-dimensional Toda lattice. Some progress toward creating the classification method was done in [9]. It was observed that any finitely generated subring of the characteristic Lie ring for the integrable case is of finite dimension. The statement was verified for a large class of the known integrable lattices.

Our interest to the Shabat's problem was stimulated by the success of the method of the hydrodynamic type reductions in the multidimensionality proposed in [5,6]. State-of-the-art for the subject and the references can be found in [16].

In the present article the lattice (1.1) is used as a touchstone for the created algorithm. Our aim is to explain the core of the method and approve its efficiency by solving a relevant classification problem.

Boundary condition of the form (1.2) imposed at two different integers $n=N_{1}$ and $n=N_{2}$ (take $N_{1}<N_{2}-1$ ) reduces the lattice (1.1) into a finite system of hyperbolic type equations (open chain)

$$
\begin{aligned}
& u_{N_{1}}=c_{1}, \\
& u_{n, x y}=\alpha\left(u_{n+1}, u_{n}, u_{n-1}\right) u_{n, x} u_{n, y}, \quad N_{1}<n<N_{2}, \\
& u_{N_{2}}=c_{2} .
\end{aligned}
$$

Initiated by the article [9], where a large class of two-dimensional lattices is discussed we use the following

Definition 1.1. We call the lattice (1.1) integrable if the hyperbolic type system (1.3) obtained from (1.1) by imposing degenerate boundary conditions is Darboux integrable for any choice of the integers $N_{1}, N_{2}$.

Recall that a system (1.3) of the hyperbolic type partial differential equations is Darboux integrable if it admits the complete set of functionally independent integrals in both of $x$ and $y$ directions. Function $I$ of a finite number of the dynamical variables $\mathbf{u}, \mathbf{u}_{x}, \mathbf{u}_{y}, \ldots$ is a $y$-integral if it satisfies the condition $D_{y} I=0$, where $D_{y}$ is the operator of the total derivative with respect to the variable $y$ and $\mathbf{u}$ is a vector with the coordinates $u_{N_{1}+1}, u_{N_{1}+2}, \ldots, u_{N_{2}-1}$ coinciding with the field variables. Since the system (1.3) is autonomous we can restrict ourselves by considering only autonomous nontrivial integrals. It can be verified that the $y$-integral does not depend on $\mathbf{u}_{y}, \mathbf{u}_{y y}, \ldots$. In what follows we are interested only on nontrivial $y$-integrals, i.e., integrals containing dependence on at least one dynamical variable $\mathbf{u}, \mathbf{u}_{x}, \ldots$ Note that currently the Darboux integrable discrete and continuous models are intensively studied (see, $[7,9,10,21,22,25,26,27,28,29])$.

We justify Definition 1.1 by the following reasoning. The problem of finding general solution to the Darboux integrable system is reduced to a problem of solving a system of the ordinary differential equations. Usually these ODE are explicitly solved. On the other hand side any solution to the considered hyperbolic system (1.3) is easily prolonged outside the interval $\left[N_{1}, N_{2}\right]$ and generates a solution of the corresponding lattice (1.1). Therefore in this case the lattice (1.1) has a large set of the explicit solutions and is definitely integrable. 
Let us briefly discuss on the content of the article. In Section 2 we recall the necessary definitions and study the main properties of the characteristic Lie ring which is a basic implement in the theory of the Darboux integrable systems. The goal of Section 3 consists in deriving some differential equations on the unknown $\alpha$ (it is reasonable to call them integrability conditions) from the finite-dimensionality property of the characteristic Lie ring. To this end we used two test sequences. In Section 4 by summarizing the integrability conditions we found the final form of the searched function $\alpha$. It is remarkable that two test sequences turned out to be enough to complete the classification. The classification result is formulated in Theorem 5.1 (see Section 5) which claims: any lattice (1.1) integrable in the sense of Definition 1.1 can be reduced by an appropriate point transformation $v=p(u)$ to the following one, found earlier in [4] and, independently, in [20]

$$
v_{n, x y}=v_{n, x} v_{n, y}\left(\frac{1}{v_{n}-v_{n-1}}-\frac{1}{v_{n+1}-v_{n}}\right) .
$$

We obtained also a new result concerned to the lattice (1.4) by proving that for any choice of the integer $N \geq 0$ the system of the hyperbolic type equations

$$
\begin{aligned}
& v_{-1}=c_{0}, \\
& v_{n, x y}=v_{n, x} v_{n, y}\left(\frac{1}{v_{n}-v_{n-1}}-\frac{1}{v_{n+1}-v_{n}}\right), \\
& v_{N+1}=c_{1}, \quad 0 \leq n \leq N
\end{aligned}
$$

admits a complete set of functionally independent $x$ - and $y$-integrals for any constant parameters $c_{0}, c_{1}$, i.e., is Darboux integrable. This fact follows immediately from Theorem 5.2 proved in Appendix A, which states that the characteristic Lie rings in both characteristic directions $x$ and $y$ for the system (1.5) are of finite dimension. In the particular case when $N=1$ for the corresponding system

$$
v_{0, x y}=v_{0, x} v_{0, y}\left(\frac{1}{v_{0}-c_{0}}-\frac{1}{v_{1}-v_{0}}\right), \quad v_{1, x y}=v_{1, x} v_{1, y}\left(\frac{1}{v_{1}-v_{0}}-\frac{1}{c_{1}-v_{1}}\right)
$$

we give the $y$ - and $x$-integrals in an explicit form

$$
\begin{aligned}
I_{1}=\frac{v_{0, x} v_{1, x}}{\left(v_{0}-c_{0}\right)\left(v_{1}-v_{0}\right)\left(c_{1}-v_{1}\right)}, & I_{2}=\frac{v_{1, x x}}{v_{1, x}}+\frac{v_{0, x}\left(v_{1}-c_{0}\right)}{\left(v_{0}-c_{0}\right)\left(v_{1}-v_{0}\right)}+\frac{2 v_{1, x}}{c_{1}-v_{1}}, \\
J_{1}=\frac{v_{0, y} v_{1, y}}{\left(v_{0}-c_{0}\right)\left(v_{1}-v_{0}\right)\left(c_{1}-v_{1}\right)}, & J_{2}=\frac{v_{1, y y}}{v_{1, y}}+\frac{v_{0, y}\left(v_{1}-c_{0}\right)}{\left(v_{0}-c_{0}\right)\left(v_{1}-v_{0}\right)}+\frac{2 v_{1, y}}{c_{1}-v_{1}} .
\end{aligned}
$$

\section{Characteristic Lie rings}

Since the lattice (1.1) is invariant under the shift of the variable $n$ we can without loss of generality take $N_{1}=-1$ and concentrate on the system

$$
\begin{aligned}
& u_{-1}=c_{0}, \\
& u_{n, x y}=\alpha_{n} u_{n, x} u_{n, y}, \quad 0 \leq n \leq N, \\
& u_{N+1}=c_{1} .
\end{aligned}
$$

Here $\alpha_{n}=\alpha\left(u_{n-1}, u_{n}, u_{n+1}\right)$. Assume that system (2.1) is Darboux integrable and that $I\left(\mathbf{u}, \mathbf{u}_{x}, \ldots\right)$ is its nontrivial integral. Let us evaluate $D_{y} I$ in the equation $D_{y} I=0$ and get due to the chain rule an equation $Y I=0$, where

$$
Y=\sum_{i=0}^{N}\left(u_{i, y} \frac{\partial}{\partial u_{i}}+f_{i} \frac{\partial}{\partial u_{i, x}}+f_{i, x} \frac{\partial}{\partial u_{i, x x}}+\cdots\right) \text {. }
$$


Here $f_{i}=\alpha_{i} u_{i, x} u_{i, y}$. Since the coefficients of the equation $Y I=0$ depend on $u_{i, y}$ while its solution $I$ does not depend on them we have a system of several linear equations for one unknown $I$

$$
Y I=0, \quad X_{j} I=0, \quad j=1, \ldots, N,
$$

with $X_{i}=\frac{\partial}{\partial u_{i, y}}$. It follows from (2.3) that for $\forall i$ the operator $Y_{i}=\left[X_{i}, Y\right]=X_{i} Y-Y X_{i}$ also annihilates $I$. Let us give the explicit form of the operator $Y_{i}$

$$
Y_{i}=\frac{\partial}{\partial u_{i}}+X_{i}\left(f_{i}\right) \frac{\partial}{\partial u_{i, x}}+X_{i}\left(D_{i} f_{i}\right) \frac{\partial}{\partial u_{i, x x}}+\cdots .
$$

Due to the relation $D_{x}^{k} f_{i}=u_{i, y} X_{i}\left(D_{x}^{k} f_{i}\right)$ we represent $(2.2)$ as

$$
Y=\sum_{i=0}^{N} u_{i, y}\left(\frac{\partial}{\partial u_{i}}+X_{i}\left(f_{i}\right) \frac{\partial}{\partial u_{i, x}}+X_{i}\left(D_{x} f_{i}\right) \frac{\partial}{\partial u_{i, x x}}+\cdots\right)=\sum_{i=0}^{N} u_{i, y} Y_{i}
$$

The last equation together with (2.3) implies $\sum_{i=0}^{N} u_{i, y} Y_{i} I=0$. Since the variables $u_{i, y}$ are independent the coefficients of this decomposition all vanish. Now we use the evident relation $\left[X_{k}, Y_{s}\right]=0$ valid for $\forall k, s$. The condition $X_{i} I=0$ is satisfied automatically. Thus we arrive at the statement: function $I$ is a $y$-integral of the system (2.1) if and only if it solves the following system of equations

$$
Y_{i} I=0 \quad \text { for } \quad i=0,1, \ldots, N
$$

Consider the set $R_{0}(y, N)$ of all multiple commutators of the characteristic vector fields $Y_{0}, Y_{1}, \ldots, Y_{N}$. Denote through $R(y, N)$ the minimal ring containing $R_{0}(y, N)$. We refer to $R(y, N)$ as the characteristic Lie ring of the system (2.1) in $y$-direction. In a similar way one can define the characteristic Lie ring in the direction of $x$. Thus we have a complete description of the set of the linear first order partial differential equations the $y$-integral should satisfy to. Now the task is to find a subset of the linearly independent equations such that all the other equations can be represented as linear combinations of those ones.

We say that the ring $R(y, N)$ is of finite dimension if there exists a finite subset $\left\{Z_{1}, Z_{2}, \ldots\right.$, $\left.Z_{L}\right\} \subset R(y, N)$ which defines a basis in $R(y, N)$ such that

1) every element $Z \in R(y, N)$ is represented in the form $Z=\lambda_{1} Z_{1}+\cdots+\lambda_{L} Z_{L}$ with the coefficients $\lambda_{1}, \ldots, \lambda_{L}$ which might depend on a finite number of the dynamical variables,

2) relation $\lambda_{1} Z_{1}+\cdots+\lambda_{L} Z_{L}=0$ implies that $\lambda_{1}=\cdots=\lambda_{L}=0$.

Let us formulate now an effective algebraic criterion (see, for instance [27, 28]) of solvability of the system (2.5).

Theorem 2.1. The system (2.1) is Darboux integrable if and only if both characteristic Lie rings $R(x, N), R(y, N)$ are of finite dimension.

Corollary 2.2. The system (2.5) has a nontrivial solution if and only if the ring $R(y, N)$ is of finite dimension.

For the sake of convenience we introduce the following notation $\operatorname{ad}_{X}(Z):=[X, Z]$. We stress that in our further study the operator $\operatorname{ad}_{D_{x}}$ plays a crucial role. Below we apply $D_{x}$ to smooth functions of the dynamical variables $\mathbf{u}, \mathbf{u}_{x}, \mathbf{u}_{x x}, \ldots$ As it was demonstrated above on this class 
of functions the operators $D_{y}$ and $Y$ coincide. Therefore relation $\left[D_{x}, D_{y}\right]=0$ immediately gives $\left[D_{x}, Y\right]=0$. Replace now $Y$ due to (2.4) and get

$$
\left[D_{x}, Y\right]=\sum_{i=0}^{N} u_{i, y}\left(\alpha_{i} u_{i, x} Y_{i}+\left[D_{x}, Y_{i}\right]\right)=0 .
$$

Since in (2.6) the variables $\left\{u_{i, y}\right\}_{i=0}^{N}$ are linearly independent, the coefficients should vanish. Consequently we have

$$
\left[D_{x}, Y_{i}\right]=-\alpha_{i} u_{i, x} Y_{i}
$$

From this formula we can easily obtain that $\operatorname{ad}_{D_{x}}: R(y, N) \rightarrow R(y, N)$. The following lemma describes the kernel of this map (see also [19])

Lemma 2.3. If the vector field

$$
Z=\sum_{i} z_{1, i} \frac{\partial}{\partial u_{i, x}}+z_{2, i} \frac{\partial}{\partial u_{i, x x}}+\cdots
$$

satisfies the condition $\left[D_{x}, Z\right]=0$ then $Z=0$.

\section{Method of the test sequences}

We call a sequence of the operators $W_{0}, W_{1}, W_{2}, \ldots$ in $R(y, N)$ a test sequence if the following condition is satisfied for $\forall m$

$$
\left[D_{x}, W_{m}\right]=\sum_{j=0}^{m} w_{j, m} W_{j}
$$

The test sequence allows one to derive integrability conditions for the hyperbolic type system (2.1) (see [10, 27, 28]). Indeed, let us assume that (2.1) is Darboux integrable. Then the ring $R(y, N)$ is of finite dimension. Therefore there exists an integer $k$ such that the operators $W_{0}, \ldots, W_{k}$ are linearly independent while the operator $W_{k+1}$ is expressed through them as follows

$$
W_{k+1}=\lambda_{k} W_{k}+\cdots+\lambda_{0} W_{0} .
$$

Let us apply the operator $\operatorname{ad}_{D_{x}}$ to both sides of (3.1). As a result we find

$$
\begin{aligned}
& \sum_{j=0}^{k} w_{j, k+1} W_{j}+w_{k+1, k+1} \sum_{j=0}^{k} \lambda_{j} W_{j} \\
& \quad=\sum_{j=0}^{k} D_{x}\left(\lambda_{j}\right) W_{j}+\lambda_{k} \sum_{j=0}^{k} w_{j, k} W_{j}+\lambda_{k-1} \sum_{j=0}^{k-1} w_{j, k-1} W_{j}+\cdots+\lambda_{0} w_{0,0} W_{0} .
\end{aligned}
$$

By collecting the coefficients before the independent operators we obtain a system of the differential equations for the coefficients $\lambda_{0}, \lambda_{1}, \ldots, \lambda_{k}$. The system is overdetermined since all of the coefficients $\lambda_{j}$ are functions of a finite number of the dynamical variables $\mathbf{u}, \mathbf{u}_{x}, \ldots$. The consistency conditions of this overdetermined system generate integrability conditions for the hyperbolic type system (2.1). For instance, collecting the coefficients before $W_{k}$ we find the first equation of the mentioned system

$$
D_{x}\left(\lambda_{k}\right)=\lambda_{k}\left(w_{k+1, k+1}-w_{k, k}\right)+w_{k, k+1},
$$

which is also overdetermined.

Below we use two different samples of the test sequences in order to find the function $\alpha_{n}$. 


\subsection{The first test sequence}

Define a sequence of the operators in $R(y, N)$ due to the recurrent formula

$$
Y_{0}, \quad Y_{1}, \quad W_{1}=\left[Y_{0}, Y_{1}\right], \quad W_{2}=\left[Y_{0}, W_{1}\right], \quad \ldots, \quad W_{k+1}=\left[Y_{0}, W_{k}\right], \quad \ldots
$$

In the case of the first two members of the sequence we have already deduced commutation relations (see (2.7) above) which are important for our further studies

$$
\left[D_{x}, Y_{0}\right]=-\alpha_{0} u_{0, x} Y_{0}, \quad\left[D_{x}, Y_{1}\right]=-\alpha_{1} u_{1, x} Y_{1}
$$

By using these two relations and applying the Jacobi identity we get immediately

$$
\left[D_{x}, W_{1}\right]=-\left(\alpha_{0} u_{0, x}+\alpha_{1} u_{1, x}\right) W_{1}-Y_{0}\left(\alpha_{1} u_{1, x}\right) Y_{1}+Y_{1}\left(\alpha_{0} u_{0, x}\right) Y_{0} .
$$

It can be proved by induction that (3.3) is really a test sequence. Moreover it is easily verified that for $k \geq 2$

$$
\left[D_{x}, W_{k}\right]=p_{k} W_{k}+q_{k} W_{k-1}+\cdots,
$$

where the factors $p_{k}, q_{k}$ are evaluated as follows

$$
p_{k}=-\left(\alpha_{1} u_{1, x}+k \alpha_{0} u_{0, x}\right), \quad q_{k}=\frac{k-k^{2}}{2} Y_{0}\left(\alpha_{0} u_{0, x}\right)-Y_{0}\left(\alpha_{1} u_{1, x}\right) k .
$$

Due to the assumption that $R(y, N)$ is of finite dimension only a finite subset of the sequence (3.3) is linearly independent. So there exists $M$ such that

$$
W_{M}=\lambda W_{M-1}+\cdots,
$$

where the operators $Y_{0}, Y_{1}, W_{1}, \ldots, W_{M-1}$ are linearly independent and the tail might contain a linear combination of the operators $Y_{0}, Y_{1}, W_{1}, \ldots, W_{M-2}$. At the moment we are not interested in that part in (3.6).

Lemma 3.1. The operators $Y_{0}, Y_{1}, W_{1}$ are linearly independent.

Proof. Assume that

$$
\lambda_{1} W_{1}+\mu_{1} Y_{1}+\mu_{0} Y_{0}=0
$$

Since the operators $Y_{0}, Y_{1}$ are of the form $Y_{0}=\frac{\partial}{\partial u_{0}}+\cdots, Y_{1}=\frac{\partial}{\partial u_{1}}+\cdots$ while $W_{1}$ does not contain summands like $\frac{\partial}{\partial u_{0}}$ and $\frac{\partial}{\partial u_{1}}$ then the factors $\mu_{1}, \mu_{0}$ vanish. If in addition $\lambda_{1} \neq 0$ then we have $W_{1}=0$. Now by applying the operator $\operatorname{ad}_{D_{x}}$ to both sides of this relation we get due to $(3.5)$ an equation

$$
Y_{0}\left(\alpha_{1} u_{1, x}\right) Y_{1}-Y_{1}\left(\alpha_{0} u_{0, x}\right) Y_{0}=0,
$$

which yields two conditions: $Y_{0}\left(\alpha_{1} u_{1, x}\right)=\alpha_{1, u_{0}} u_{1, x}=0$ and $Y_{1}\left(\alpha_{0} u_{0, x}\right)=\alpha_{0, u_{1}} u_{0, x}=0$. Those equalities contradict our assumption that $\frac{\partial \alpha\left(u_{n+1}, u_{n}, u_{n-1}\right)}{\partial u_{n \pm 1}} \neq 0$. Lemma is proved.

Lemma 3.2. If the expansion (3.6) holds then

$$
\alpha\left(u_{1}, u_{0}, u_{-1}\right)=\frac{P^{\prime}\left(u_{0}\right)}{P\left(u_{0}\right)+Q\left(u_{-1}\right)}+\frac{1}{M-1} \frac{Q^{\prime}\left(u_{0}\right)}{P\left(u_{1}\right)+Q\left(u_{0}\right)}-c_{1}\left(u_{0}\right) .
$$


Proof. It is easy to check that equation (3.2) for the case of the sequence (3.3) takes the following form

$$
D_{x}(\lambda)=-\alpha_{0} u_{0, x} \lambda-\frac{M(M-1)}{2} Y_{0}\left(\alpha_{0} u_{0, x}\right)-M Y_{0}\left(\alpha_{1} u_{1, x}\right) .
$$

We simplify the formula (3.7) due to the relations

$$
\begin{aligned}
& Y_{0}\left(\alpha_{0} u_{0, x}\right)=\left(\frac{\partial}{\partial u_{0}}+\alpha_{0} u_{0, x} \frac{\partial}{\partial u_{0, x}}\right) \alpha_{0} u_{0, x}=\left(\alpha_{0, u_{0}}+\alpha_{0}^{2}\right) u_{0 x}, \\
& Y_{0}\left(\alpha_{1} u_{1, x}\right)=\alpha_{1, u_{0}} u_{1, x} .
\end{aligned}
$$

A simple analysis of the equation (3.7) gives that $\lambda=\lambda\left(u_{0}, u_{1}\right)$. Therefore (3.7) gives rise to the equation

$$
\lambda_{u_{0}} u_{0, x}+\lambda_{u_{1}} u_{1, x}=-\left(\alpha \lambda+\frac{M(M-1)}{2}\left(\alpha_{0, u_{0}}+\alpha_{0}^{2}\right)\right) u_{0, x}-M \alpha_{1, u_{0}} u_{1, x} .
$$

By comparing the coefficients before the independent variables $u_{0, x}, u_{1, x}$ we deduce an overdetermined system of the differential equations for $\lambda$

$$
\lambda_{u_{0}}=-\alpha_{0} \lambda-\frac{M(M-1)}{2}\left(\alpha_{0, u_{0}}+\alpha_{0}^{2}\right), \quad \lambda_{u_{1}}=-M \alpha_{1, u_{0}} .
$$

Let us derive and investigate the consistency conditions of the system (3.8). We differentiate the first equation with respect to $u_{-1}$ and find

$$
\lambda=-\frac{M(M-1)}{2} \frac{\alpha_{0, u_{0} u_{-1}}+2 \alpha_{0} \alpha_{0, u_{-1}}}{\alpha_{0, u_{-1}}} .
$$

Since $\lambda_{u_{-1}}=0$ we have

$$
\left(\log \alpha_{0, u_{-1}}\right)_{u_{0} u_{-1}}+2 \alpha_{0, u_{-1}}=0 .
$$

Now we introduce a new variable $z$ due to the relation $\alpha_{0, u_{-1}}=-\frac{1}{2} e^{z}$ and reduce (3.10) to the Liouville equation $z_{u_{0} u_{-1}}=e^{z}$ for which we have the general solution

$$
e^{z}=\frac{2 P^{\prime}\left(u_{0}\right) Q^{\prime}\left(u_{-1}\right)}{\left(P\left(u_{0}\right)+Q\left(u_{-1}\right)\right)^{2}},
$$

where $P\left(u_{0}\right)$ and $Q\left(u_{-1}\right)$ are arbitrary differentiable functions. Thus for $\alpha_{0}$ we can obtain the following explicit expression

$$
\alpha_{0}=-\frac{1}{2} \int e^{z} d u_{-1}=\frac{P^{\prime}\left(u_{0}\right)}{P\left(u_{0}\right)+Q\left(u_{-1}\right)}+H\left(u_{0}, u_{1}\right),
$$

where $H\left(u_{0}, u_{1}\right)$ is to be determined. Now we can find $\lambda$ from the second equation in (3.8)

$$
\lambda=-M \int \alpha_{1, u_{0}} d u_{1}=-M \frac{Q^{\prime}\left(u_{0}\right)}{P\left(u_{1}\right)+Q\left(u_{0}\right)}+M c\left(u_{0}\right) .
$$

Let us specify $H\left(u_{0}, u_{1}\right)$ by replacing in (3.9) $\alpha_{0}$ and $\lambda$ in virtue of (3.11), (3.12). As a result we obtain

$$
H\left(u_{0}, u_{1}\right)=\frac{1}{M-1} \frac{Q^{\prime}\left(u_{0}\right)}{P\left(u_{1}\right)+Q\left(u_{0}\right)}-\frac{1}{M-1} c\left(u_{0}\right)-\frac{1}{2} \frac{P^{\prime \prime}\left(u_{0}\right)}{P^{\prime}\left(u_{0}\right)} .
$$

Summarizing the reasonings we can conclude that

$$
\alpha\left(u_{1}, u_{0}, u_{-1}\right)=\frac{P^{\prime}\left(u_{0}\right)}{P\left(u_{0}\right)+Q\left(u_{-1}\right)}+\frac{1}{M-1} \frac{Q^{\prime}\left(u_{0}\right)}{P\left(u_{1}\right)+Q\left(u_{0}\right)}-c_{1}\left(u_{0}\right),
$$

where the functions of one variable $P\left(u_{0}\right), Q\left(u_{0}\right), c_{1}\left(u_{0}\right)=\frac{1}{M-1} c\left(u_{0}\right)+\frac{1}{2} \frac{P^{\prime \prime}\left(u_{0}\right)}{P^{\prime}\left(u_{0}\right)}$ and the integer $M$ are to be found. 
The next step requires some additional integrability conditions. In what follows we derive them by constructing another test sequence.

\subsection{The second test sequence}

Now we concentrate on a test sequence generated by the operators $Y_{0}, Y_{1}, Y_{2}$ and their multiple commutators. It is more complicated than the previous sequence

$$
\begin{aligned}
& Z_{0}=Y_{0}, \quad Z_{1}=Y_{1}, \quad Z_{2}=Y_{2}, \quad Z_{3}=\left[Y_{1}, Y_{0}\right], \quad Z_{4}=\left[Y_{2}, Y_{1}\right], \\
& Z_{5}=\left[Y_{2}, Z_{3}\right], \quad Z_{6}=\left[Y_{1}, Z_{3}\right], \quad Z_{7}=\left[Y_{1}, Z_{4}\right], \quad Z_{8}=\left[Y_{1}, Z_{5}\right] .
\end{aligned}
$$

The members $Z_{m}$ of the sequence for $m>8$ are defined due to the recurrence $Z_{m}=\left[Y_{1}, Z_{m-3}\right]$. Note that it is the simplest test sequence generated by the iterations of the map $Z \rightarrow\left[Y_{1}, Z\right]$ which contains the operator $\left[Y_{2},\left[Y_{1}, Y_{0}\right]\right]=Z_{5}$.

Lemma 3.3. Operators $Z_{0}, Z_{1}, \ldots, Z_{5}$ constitute a linearly independent set.

Proof. Firstly we note that the operators $Z_{0}, Z_{1}, \ldots, Z_{4}$ are linearly independent. It can be verified by using reasonings similar to those from the proof of Lemma 3.2. We prove the lemma by contradiction. Assume that

$$
Z_{5}=\sum_{j=0}^{4} \lambda_{j} Z_{j}
$$

Now we specify the action of the operator $\operatorname{ad}_{D_{x}}$ on the operators $Z_{i}$. For $i=0,1,2$ it is obtained from the relation

$$
\left[D_{x}, Y_{i}\right]=-\alpha_{i} u_{i, x} Y_{i}
$$

Recall that $\alpha_{i}=\alpha\left(u_{i-1}, u_{i}, u_{i+1}\right)$. For $i=3,4,5$ we have

$$
\begin{aligned}
& {\left[D_{x}, Z_{3}\right]=-\left(a_{1}+a_{0}\right) Z_{3}+\cdots,} \\
& {\left[D_{x}, Z_{4}\right]=-\left(a_{2}+a_{1}\right) Z_{4}+\cdots} \\
& {\left[D_{x}, Z_{5}\right]=-\left(a_{0}+a_{1}+a_{2}\right) Z_{5}+Y_{0}\left(a_{1}\right) Z_{4}-Y_{2}\left(a_{1}\right) Z_{3}+\cdots}
\end{aligned}
$$

Here $a_{i}=\alpha_{i} u_{i, x}$. Let us apply the operator $\operatorname{ad}_{D_{x}}$ to both sides of (3.15) and obtain

$$
\begin{gathered}
-\left(a_{0}+a_{1}+a_{2}\right)\left(\lambda_{4} Z_{4}+\lambda_{3} Z_{3}+\cdots\right)+Y_{0}\left(a_{1}\right) Z_{4}-Y_{2}\left(a_{1}\right) Z_{3}+\cdots \\
=\lambda_{4, x} Z_{4}+\lambda_{3, x} Z_{3}-\lambda_{4}\left(a_{1}+a_{2}\right) Z_{4}-\lambda_{3}\left(a_{0}+a_{1}\right) Z_{3}+\cdots
\end{gathered}
$$

By comparing the coefficients before $Z_{4}$ in (3.16) we obtain the following equation

$$
\lambda_{4, x}=-\alpha_{0} u_{0, x} \lambda_{4}-\alpha_{1, u_{0}} u_{1, x} .
$$

A simple analysis of the equation (3.17) shows that $\lambda=\lambda\left(u_{0}, u_{1}\right)$. Hence the equation (3.17) splits down into two equations $\lambda_{4, u_{0}}=-\alpha_{0} \lambda_{4}$ and $\lambda_{4, u_{1}}=-\alpha_{1, u_{0}}$. The former shows that $\lambda_{4}=0$. Indeed if $\lambda_{4} \neq 0$ then we obtain an expression for $\alpha_{0}: \alpha_{0}=-\left(\log \lambda_{4}\right)_{u_{0}}$ which shows that $\left(\alpha_{0}\right)_{u_{-1}}=0$. It contradicts the assumption that $\alpha\left(u_{1}, u_{0}, u_{-1}\right)$ depends essentially on $u_{1}$ and $u_{-1}$, therefore $\lambda_{4}=0$. Then (3.17) implies $\alpha_{1, u_{0}}=0$ and it leads again to a contradiction.

Turn back to the sequence (3.14). For the further study it is necessary to specify the action of the operator $\operatorname{ad}_{D_{x}}$ on the members of this sequence. It is convenient to divide the sequence into three subsequences and study them separately $\left\{Z_{3 m}\right\},\left\{Z_{3 m+1}\right\}$, and $\left\{Z_{3 m+2}\right\}$. 
Lemma 3.4. Action of the operator $\operatorname{ad}_{D_{x}}$ on the sequence (3.14) is given by the following relations

$$
\begin{aligned}
{\left[D_{x}, Z_{3 m}\right]=} & -\left(\alpha_{0} u_{0, x}+m \alpha_{1} u_{1, x}\right) Z_{3 m} \\
+ & \left(\frac{m-m^{2}}{2} Y_{1}\left(\alpha_{1} u_{1, x}\right)-m Y_{1}\left(\alpha_{0} u_{0, x}\right)\right) Z_{3 m-3}+\cdots, \\
{\left[D_{x}, Z_{3 m+1}\right]=} & -\left(\alpha_{2} u_{2, x}+m \alpha_{1} u_{1, x}\right) Z_{3 m+1} \\
& +\left(\frac{m-m^{2}}{2} Y_{1}\left(\alpha_{1} u_{1, x}\right)-m Y_{1}\left(\alpha_{2} u_{2, x}\right)\right) Z_{3 m-2}+\cdots, \\
{\left[D_{x}, Z_{3 m+2}\right]=} & -\left(\alpha_{0} u_{0, x}+m \alpha_{1} u_{1, x}+\alpha_{2} u_{2, x}\right) Z_{3 m+2}+Y_{0}\left(\alpha_{1} u_{1, x}\right) Z_{3 m+1}+Y_{2}\left(\alpha_{1} u_{1, x}\right) Z_{3 m} \\
& -(m-1)\left(\frac{m}{2} Y_{1}\left(\alpha_{1} u_{1, x}\right)+Y_{1}\left(\alpha_{0} u_{0, x}+\alpha_{2} u_{2, x}\right)\right) Z_{3 m-1}+\cdots .
\end{aligned}
$$

Lemma 3.4 is easily proved by induction. Since the proof is quite technical we omit it.

Theorem 3.5. Assume that $Z_{3 k+2}$ is represented as a linear combination

$$
Z_{3 k+2}=\lambda_{k} Z_{3 k+1}+\mu_{k} Z_{3 k}+\nu_{k} Z_{3 k-1}+\cdots
$$

of the previous members of the sequence (3.14) and neither of the operators $Z_{3 j+2}$ with $j<k$ is a linear combination of $Z_{s}$ with $s<3 j+2$. Then the coefficient $\nu_{k}$ is a solution to the equation

$$
D_{x}\left(\nu_{k}\right)=-\alpha_{1} u_{1, x} \nu_{k}-\frac{k(k-1)}{2} Y_{1}\left(\alpha_{1} u_{1, x}\right)-(k-1) Y_{1}\left(\alpha_{0} u_{0, x}+\alpha_{2} u_{2, x}\right) .
$$

Lemma 3.6. Suppose that all of the conditions of the theorem are satisfied. In addition assume that the operator $Z_{3 k}$ (operator $Z_{3 k+1}$ ) is linearly expressed in terms of the operator $Z_{i}$ with $i<3 k$. Then in this decomposition the coefficient before $Z_{3 k-1}$ vanishes.

Proof. Assume in contrary that $\lambda \neq 0$ in the formula

$$
Z_{3 k}=\lambda Z_{3 k-1}+\cdots .
$$

Let us apply $\operatorname{ad}_{D_{x}}$ to (3.20). As a result we find due to Lemma 3.4

$$
\begin{aligned}
& -\left(\alpha_{0} u_{0, x}+k \alpha_{1} u_{1, x}\right) \lambda Z_{3 k-1}+\cdots \\
& \quad=D_{x}(\lambda) Z_{3 k-1}-\lambda\left(\alpha_{0} u_{0, x}+(k-1) \alpha_{1} u_{1, x}+\alpha_{2} u_{2, x}\right) Z_{3 k-1}+\cdots .
\end{aligned}
$$

Collect the coefficients before $Z_{3 k-1}$ and obtain an equation the coefficient $\lambda$ must satisfy to

$$
D_{x}(\lambda)=\lambda\left(\alpha_{2} u_{2, x}-\alpha_{1} u_{1, x}\right) .
$$

Due to our assumption above $\lambda$ does not vanish and hence

$$
D_{x}(\log \lambda)=\alpha_{2} u_{2, x}-\alpha_{1} u_{1, x} .
$$

Since $\lambda$ depends on a finite number of the dynamical variables then due to equation (3.22) $\lambda$ might depend only on $u_{1}$ and $u_{2}$. Therefore (3.21) yields

$$
(\log \lambda)_{u_{1}} u_{1, x}+(\log \lambda)_{u_{2}} u_{2, x}=\alpha_{2} u_{2, x}-\alpha_{1} u_{1, x} .
$$

The variables $u_{1, x}, u_{2, x}$ are independent, so the last equation implies $\alpha_{1}=-(\log \lambda)_{u_{1}}, \alpha_{2}=$ $(\log \lambda)_{u_{2}}$. Thus $\alpha_{1}=\alpha_{1}\left(u_{1}, u_{2}\right)$ depends only on $u_{1}, u_{2}$. It contradicts our assumption that $\alpha_{1}$ depends essentially on $u_{0}$. The contradiction shows that assumption $\lambda \neq 0$ is not true. That completes the proof. 
Now in order to prove Theorem 3.5 we apply the operator $\operatorname{ad}_{D_{x}}$ to both sides of (3.18) and then simplify due to the relation from Lemma 3.4. Comparison of the coefficients before $Z_{3 k-1}$ implies equation (3.19).

Let us find the explicit expressions for the coefficients of the equation (3.19)

$$
Y_{1}\left(\alpha_{0} u_{0, x}\right)=\alpha_{0, u_{1}} u_{0, x}, \quad Y_{1}\left(\alpha_{2} u_{2, x}\right)=\alpha_{2, u_{1}} u_{2, x}, \quad Y_{1}\left(\alpha_{1} u_{1, x}\right)=\left(\alpha_{1, u_{1}}+\alpha_{1}^{2}\right) u_{1, x}
$$

and substitute them into (3.19)

$$
D_{x}\left(\nu_{k}\right)=-\alpha_{1} \nu_{k} u_{1, x}-\frac{k(k-1)}{2}\left(\alpha_{1, u_{1}}+\alpha_{1}^{2}\right) u_{1, x}-(k-1)\left(\alpha_{0, u_{1}} u_{x}+\alpha_{2, u_{1}} u_{2, x}\right) .
$$

A simple analysis of (3.23) convinces that $\nu_{k}$ might depend only on the variables $u_{0}, u_{1}, u_{2}$. Therefore

$$
D_{x}\left(\nu_{k}\right)=\nu_{k, u_{0}} u_{0, x}+\nu_{k, u_{1}} u_{1, x}+\nu_{k, u_{2}} u_{2, x} .
$$

From the equations $(3.23),(3.24)$ we obtain a system of the equations for the coefficient $\nu_{k}$

$$
\begin{aligned}
& \nu_{k, u_{0}}=-(k-1) \alpha_{0, u_{1}}, \\
& \nu_{k, u_{1}}=-\alpha_{1} \nu_{k}-\frac{k(k-1)}{2}\left(\alpha_{1, u_{1}}+\alpha_{1}^{2}\right), \\
& \nu_{k, u_{2}}=-(k-1) \alpha_{2, u_{1}} .
\end{aligned}
$$

Substitute the preliminary expression for the function $\alpha$ given by the formula (3.13) into the equation (3.25) and get

$$
\nu_{k, u_{0}}=\frac{k-1}{M-1} \frac{P^{\prime}\left(u_{1}\right) Q^{\prime}\left(u_{0}\right)}{\left(P\left(u_{1}\right)+Q\left(u_{0}\right)\right)^{2}} .
$$

Integration of the latter with respect to $u_{0}$ yields

$$
\nu_{k}=-\frac{k-1}{M-1} \frac{P^{\prime}\left(u_{1}\right)}{P\left(u_{1}\right)+Q\left(u_{0}\right)}+H\left(u_{1}, u_{2}\right) .
$$

Since $\nu_{k, u_{2}}=H_{u_{2}}$ the equation (3.27) gives rise to the relation

$$
H_{u_{2}}=(k-1) \frac{P^{\prime}\left(u_{2}\right) Q^{\prime}\left(u_{1}\right)}{\left(P\left(u_{2}\right)+Q\left(u_{1}\right)\right)^{2}} .
$$

Now by integration we obtain an explicit formula for $H$

$$
H=-(k-1)\left(\frac{Q^{\prime}\left(u_{1}\right)}{P\left(u_{2}\right)+Q\left(u_{1}\right)}+A\left(u_{1}\right)\right)
$$

which produces

$$
\nu_{k}=-(k-1)\left(\frac{1}{M-1} \frac{P^{\prime}\left(u_{1}\right)}{P\left(u_{1}\right)+Q\left(u_{0}\right)}+\frac{Q^{\prime}\left(u_{1}\right)}{P\left(u_{2}\right)+Q\left(u_{1}\right)}+A\left(u_{1}\right)\right) .
$$


Let us substitute the values of $\alpha$ and $\nu_{k}$ found into the equation (3.26). We get a huge equation

$$
\begin{aligned}
-\frac{(k-1)}{M-} & \left(\frac{P^{\prime \prime}\left(u_{1}\right)}{P\left(u_{1}\right)+Q\left(u_{0}\right)}-\frac{P^{\prime 2}\left(u_{1}\right)}{\left(P\left(u_{1}\right)+Q\left(u_{0}\right)\right)^{2}}\right) \\
& -(k-1)\left(\frac{Q^{\prime \prime}\left(u_{1}\right)}{P\left(u_{2}\right)+Q\left(u_{1}\right)}-\frac{Q^{\prime 2}\left(u_{1}\right)}{\left(P\left(u_{2}\right)+Q\left(u_{1}\right)\right)^{2}}+A^{\prime}\left(u_{1}\right)\right) \\
= & (k-1)\left(\frac{P^{\prime}\left(u_{1}\right)}{P\left(u_{1}\right)+Q\left(u_{0}\right)}+\frac{1}{M-1} \frac{Q^{\prime}\left(u_{1}\right)}{P\left(u_{2}\right)+Q\left(u_{1}\right)}-c_{1}\left(u_{1}\right)\right) \\
& \times\left(\frac{1}{M-1} \frac{P^{\prime}\left(u_{1}\right)}{P\left(u_{1}\right)+Q\left(u_{0}\right)}+\frac{Q^{\prime}\left(u_{1}\right)}{P\left(u_{2}\right)+Q\left(u_{1}\right)}+A\left(u_{1}\right)\right) \\
& -\frac{k(k-1)}{2}\left(\frac{P^{\prime \prime}\left(u_{1}\right)}{P\left(u_{1}\right)+Q\left(u_{0}\right)}+\frac{1}{M-1} \frac{Q^{\prime \prime}\left(u_{1}\right)}{P\left(u_{2}\right)+Q\left(u_{1}\right)}\right. \\
& -\frac{1}{M-1} \frac{Q^{\prime 2}\left(u_{1}\right)}{\left(P\left(u_{2}\right)+Q\left(u_{1}\right)\right)^{2}}+\frac{1}{M-1} \frac{2 Q^{\prime}\left(u_{1}\right) P^{\prime}\left(u_{1}\right)}{\left(P\left(u_{1}\right)+Q\left(u_{0}\right)\right)\left(P\left(u_{2}\right)+Q\left(u_{1}\right)\right)} \\
& +\frac{1}{(M-1)^{2}} \frac{Q^{\prime 2}\left(u_{1}\right)}{\left(P\left(u_{2}\right)+Q\left(u_{1}\right)\right)^{2}} \\
& \left.-c_{1}^{\prime}\left(u_{1}\right)-2 c_{1}\left(u_{1}\right)\left(\frac{P^{\prime}\left(u_{1}\right)}{P\left(u_{1}\right)+Q\left(u_{0}\right)}+\frac{1}{M-1} \frac{Q^{\prime}\left(u_{1}\right)}{P\left(u_{2}\right)+Q\left(u_{1}\right)}\right)+c_{1}^{2}\left(u_{1}\right)\right) .
\end{aligned}
$$

Evidently due to our assumption $\frac{\partial}{\partial u_{1}} \alpha\left(u_{1}, u_{0}, u_{-1}\right) \neq 0, \frac{\partial}{\partial u_{-1}} \alpha\left(u_{1}, u_{0}, u_{-1}\right) \neq 0$ the functions $P^{\prime}\left(u_{2}\right)$ and $Q^{\prime}\left(u_{0}\right)$ do not vanish. Therefore the variables

$$
\frac{Q^{\prime 2}\left(u_{1}\right)}{\left(P\left(u_{2}\right)+Q\left(u_{1}\right)\right)^{2}}, \quad \frac{P^{\prime 2}\left(u_{1}\right)}{\left(P\left(u_{1}\right)+Q\left(u_{0}\right)\right)^{2}}, \quad \frac{P^{\prime}\left(u_{1}\right) Q^{\prime}\left(u_{1}\right)}{\left(P\left(u_{1}\right)+Q\left(u_{0}\right)\right)\left(P\left(u_{2}\right)+Q\left(u_{1}\right)\right)}
$$

are independent. By gathering the coefficients before these variables in (3.28) we get a system of two equations

$$
\left(1-\frac{1}{M-1}\right)\left(1-\frac{k}{2(M-1)}\right)=0, \quad 1+\frac{1}{(M-1)^{2}}=\frac{k}{M-1} .
$$

There are two solutions to the system (3.29): $M=0, k=-2$ and $M=2, k=2$. The former does not fit since $k$ should be positive, so we have the only possibility $M=2, k=2$. This finishes the proof of Theorem 3.5.

\section{Finding the functions $P, Q$ and $c_{1}$}

In this section we specify the function $\alpha$ given by (3.13). For this aim we should consider expansions (3.6), (3.18) using the fact that $M=2, k=2$.

Let us rewrite the expansion (3.6) in the complete form

$$
W_{2}=\lambda W_{1}+\sigma Y_{1}+\delta Y_{0}
$$

Theorem 4.1. Expansion (4.1) holds if and only if the function $\alpha$ in (1.1) is of the following form

$$
\alpha\left(u_{n+1}, u_{n}, u_{n-1}\right)=\frac{P^{\prime}\left(u_{n}\right)}{P\left(u_{n}\right)+Q\left(u_{n-1}\right)}+\frac{Q^{\prime}\left(u_{n}\right)}{P\left(u_{n+1}\right)+Q\left(u_{n}\right)}-\frac{1}{2}\left(\log Q^{\prime}\left(u_{n}\right) P^{\prime}\left(u_{n}\right)\right)^{\prime},
$$

where the functions $P\left(u_{n}\right), Q\left(u_{n}\right)$ are connected with each other by the differential constraint

$$
-3 Q^{\prime \prime 2} P^{\prime 2}-2 P^{\prime \prime \prime} P^{\prime} Q^{\prime 2}+3 P^{\prime \prime 2} Q^{\prime 2}+2 P^{\prime 2} Q^{\prime \prime \prime} Q^{\prime}=0 .
$$


Proof. Firstly by using relations (3.4), (3.5) and applying the Jacobi identity we get

$$
\left[D_{x}, W_{2}\right]=-\left(2 a_{0}+a_{1}\right) W_{2}-Y_{0}\left(a_{0}+2 a_{1}\right) W_{1}+\left(2 Y_{0} Y_{1}\left(a_{0}\right)-Y_{1} Y_{0}\left(a_{0}\right)\right) Y_{0}-Y_{0} Y_{0}\left(a_{1}\right) Y_{1} .
$$

Evidently only one summand in (4.1) contains the term $\frac{\partial}{\partial u_{1}}$, namely $\sigma Y_{1}$, and only one summand contains the term $\frac{\partial}{\partial u_{0}}$, namely $\delta Y_{0}$. Hence $\sigma=0, \delta=0$ and we have

$$
W_{2}=\lambda W_{1} \text {. }
$$

Now by applying the operator $\operatorname{ad}_{D_{x}}$ to both sides of this relation we obtain

$$
\begin{gathered}
-\left(2 a_{0}+a_{1}\right) W_{2}-Y_{0}\left(a_{0}+2 a_{1}\right) W_{1}+\left(2 Y_{0} Y_{1}\left(a_{0}\right)-Y_{1} Y_{0}\left(a_{0}\right)\right) Y_{0}-Y_{0} Y_{0}\left(a_{1}\right) Y_{1} \\
=D_{x}(\lambda) W_{1}+\lambda\left(-\left(a_{0}+a_{1}\right) W_{1}+Y_{1}\left(a_{0}\right) Y_{0}-Y_{0}\left(a_{1}\right) Y_{1}\right) .
\end{gathered}
$$

Collecting the coefficients before $W_{2}, W_{1}, Y_{1}$, and $Y_{0}$ we find the following system

$$
\begin{aligned}
& D_{x}(\lambda)=-a_{0} \lambda-Y_{0}\left(a_{0}+2 a_{1}\right), \\
& -Y_{0} Y_{0}\left(a_{1}\right)=-\lambda Y_{0}\left(a_{1}\right), \\
& 2 Y_{0} Y_{1}\left(a_{0}\right)-Y_{1} Y_{0}\left(a_{0}\right)=\lambda Y_{1}\left(a_{0}\right) .
\end{aligned}
$$

Setting $M=2$ in (3.7) we obtain equation (4.4). The overdetermined system (3.8) takes the form

$$
\begin{aligned}
& \lambda_{u_{0}}=-\alpha_{0} \lambda-\left(\alpha_{0, u_{0}}+\alpha_{0}^{2}\right), \\
& \lambda_{u_{1}}=-2 \alpha_{1, u_{0}} .
\end{aligned}
$$

Thus

$$
\begin{aligned}
& \lambda=-2 \frac{Q^{\prime}\left(u_{0}\right)}{P\left(u_{1}\right)+Q\left(u_{0}\right)}+2 c\left(u_{0}\right), \\
& \alpha\left(u_{1}, u_{0}, u_{-1}\right)=\frac{P^{\prime}\left(u_{0}\right)}{P\left(u_{0}\right)+Q\left(u_{-1}\right)}+\frac{Q^{\prime}\left(u_{0}\right)}{P\left(u_{1}\right)+Q\left(u_{0}\right)}-\frac{1}{2} \frac{P^{\prime \prime}\left(u_{0}\right)}{P^{\prime}\left(u_{0}\right)}-c\left(u_{0}\right) .
\end{aligned}
$$

We rewrite (4.5), (4.6) due to the relations

$$
\begin{aligned}
& Y_{0}\left(a_{0}\right)=\left(\frac{\partial}{\partial u_{0}}+\alpha_{0} u_{0, x} \frac{\partial}{\partial u_{0, x}}+\cdots\right)\left(\alpha_{0} u_{0, x}\right)=\left(\alpha_{0, u_{0}}+\alpha_{0}^{2}\right) u_{0, x}, \\
& Y_{0}\left(a_{1}\right)=\left(\frac{\partial}{\partial u_{0}}+\alpha_{0} u_{0, x} \frac{\partial}{\partial u_{0, x}}+\cdots\right)\left(\alpha_{1} u_{1, x}\right)=\alpha_{1, u_{0}} u_{1, x}, \\
& Y_{0}\left(a_{0}+2 a_{1}\right)=Y_{0}\left(a_{0}\right)+2 Y_{0}\left(a_{1}\right)=\left(\alpha_{0, u_{0}}+\alpha_{0}^{2}\right) u_{0, x}+2 \alpha_{1, u_{0}} u_{1, x}, \\
& Y_{0} Y_{0}\left(a_{1}\right)=\left(\frac{\partial}{\partial u_{0}}+\alpha_{0} u_{0, x} \frac{\partial}{\partial u_{0, x}}+\cdots\right)\left(\alpha_{1, u_{0}} u_{1, x}\right)=\alpha_{1, u_{0} u_{0}} u_{1, x}, \\
& Y_{1}\left(a_{0}\right)=\left(\frac{\partial}{\partial u_{1}}+\alpha_{1} u_{1, x} \frac{\partial}{\partial u_{1, x}}+\cdots\right)\left(\alpha_{0} u_{0, x}\right)=\alpha_{0, u_{1}} u_{0, x}, \\
& Y_{0} Y_{1}\left(a_{0}\right)=\left(\frac{\partial}{\partial u_{0}}+\alpha_{0} u_{0, x} \frac{\partial}{\partial u_{0, x}}+\cdots\right)\left(\alpha_{0, u_{1}} u_{0, x}\right)=\left(\alpha_{0, u_{0} u_{1}}+\alpha_{0} \alpha_{0, u_{1}}\right) u_{x}, \\
& Y_{1} Y_{0}\left(a_{0}\right)=\left(\frac{\partial}{\partial u_{1}}+\alpha_{1} u_{1, x} \frac{\partial}{\partial u_{1, x}}+\cdots\right)\left(\left(\alpha_{0, u_{0}}+\alpha_{0}^{2}\right) u_{0, x}\right)=\left(\alpha_{0, u_{0} u_{1}}+2 \alpha_{0} \alpha_{0, u_{1}}\right) u_{0, x}
\end{aligned}
$$

as follows

$$
\alpha_{1, u_{0} u_{0}}=\lambda \alpha_{1, u_{0}}, \quad \alpha_{0, u_{0} u_{1}}=\lambda \alpha_{0, u_{1}} .
$$


We substitute (4.8), (4.9) into (4.10) and find that $c\left(u_{0}\right)=\frac{1}{2} \frac{Q^{\prime \prime}\left(u_{0}\right)}{Q^{\prime}\left(u_{0}\right)}$. So we find that functions (4.8), (4.9) are given by

$$
\begin{aligned}
& \lambda_{(R)}=\lambda\left(u_{0}, u_{1}\right)=-2 \frac{Q^{\prime}\left(u_{0}\right)}{P\left(u_{1}\right)+Q\left(u_{0}\right)}+\frac{Q^{\prime \prime}\left(u_{0}\right)}{Q^{\prime}\left(u_{0}\right)}, \\
& \alpha\left(u_{1}, u_{0}, u_{-1}\right)=\frac{P^{\prime}\left(u_{0}\right)}{P\left(u_{0}\right)+Q\left(u_{-1}\right)}+\frac{Q^{\prime}\left(u_{0}\right)}{P\left(u_{1}\right)+Q\left(u_{0}\right)}-\frac{1}{2} \frac{P^{\prime \prime}\left(u_{0}\right)}{P^{\prime}\left(u_{0}\right)}-\frac{1}{2} \frac{Q^{\prime \prime}\left(u_{0}\right)}{Q^{\prime}\left(u_{0}\right)} .
\end{aligned}
$$

Substituting (4.11), (4.12) into (4.7) we obtain that the functions $P, Q$ must satisfy the equality

$$
-3 Q^{\prime \prime 2} P^{\prime 2}-2 P^{\prime \prime \prime} P^{\prime} Q^{\prime 2}+3 P^{\prime \prime 2} Q^{\prime 2}+2 P^{\prime 2} Q^{\prime \prime \prime} Q^{\prime}=0 .
$$

Thus we have proved that if the expansion (3.6) holds then it should be of the form

$$
W_{2}=\lambda_{(R)} W_{1}
$$

Or the same

$$
\left[Y_{0},\left[Y_{1}, Y_{0}\right]\right]=\lambda_{(R)}\left[Y_{1}, Y_{0}\right] .
$$

Let us define a sequence of the operators in $R(y, N)$ due to the following recurrent formula

$$
Y_{0}, \quad Y_{1}, \quad \tilde{W}_{1}=\left[Y_{1}, Y_{0}\right], \quad \tilde{W}_{2}=\left[Y_{1}, W_{1}\right], \quad \ldots, \quad \tilde{W}_{k+1}=\left[Y_{1}, \tilde{W}_{k}\right], \quad \ldots
$$

It slightly differs from (3.3) and can be studied in a similar way. We can easily check that the conditions (4.2), (4.3) provide the representation

$$
\tilde{W}_{2}=\lambda_{(L)} \tilde{W}_{1}
$$

Or the same

$$
\left[Y_{1},\left[Y_{1}, Y_{0}\right]\right]=\lambda_{(L)}\left[Y_{1}, Y_{0}\right]
$$

with the coefficient

$$
\lambda_{(L)}=-\frac{2 P^{\prime}\left(u_{1}\right)}{P\left(u_{1}\right)+Q\left(u_{0}\right)}+\frac{P^{\prime \prime}\left(u_{1}\right)}{P^{\prime}\left(u_{1}\right)} .
$$

Let us consider expansion (3.18) setting $k=2$,

$$
Z_{8}=\lambda Z_{7}+\mu Z_{6}+\nu Z_{5}+\rho Z_{4}+\kappa Z_{3}+\sigma Z_{2}+\delta Z_{1}+\eta Z_{0}
$$

Theorem 4.2. Expansions (4.1), (4.14) hold if and only if the function $\alpha$ in (1.1) is of one of the forms

$$
\begin{aligned}
\alpha_{0}= & \alpha\left(u_{1}, u_{0}, u_{-1}\right)=\frac{P^{\prime}\left(u_{0}\right)}{P\left(u_{0}\right)+c_{1} P\left(u_{-1}\right)+c_{2}}+\frac{c_{1} P^{\prime}\left(u_{0}\right)}{P\left(u_{1}\right)+c_{1} P\left(u_{0}\right)+c_{2}}-\frac{P^{\prime \prime}\left(u_{0}\right)}{P^{\prime}\left(u_{0}\right)}, \\
\alpha_{0}= & \alpha\left(u_{1}, u_{0}, u_{-1}\right)=\frac{c_{3} r\left(u_{-1}\right) r^{\prime}\left(u_{0}\right)}{c_{3} r\left(u_{0}\right) r\left(u_{-1}\right)+c_{4} r\left(u_{-1}\right)-c_{1}+c_{2} r\left(u_{-1}\right)} \\
& +\frac{c_{1} r^{\prime}\left(u_{0}\right)}{r\left(u_{0}\right)\left(c_{3} r\left(u_{1}\right) r\left(u_{0}\right)+c_{4} r\left(u_{0}\right)-c_{1}+c_{2} r\left(u_{0}\right)\right)}-\frac{r^{\prime \prime}\left(u_{0}\right) r\left(u_{0}\right)-r^{\prime 2}\left(u_{0}\right)}{r\left(u_{0}\right) r^{\prime}\left(u_{0}\right)}
\end{aligned}
$$

where $P\left(u_{0}\right)$ and $r\left(u_{0}\right)$ are arbitrary smooth functions, $c_{1} \neq 0, c_{3} \neq 0, c_{2}$, and $c_{4}$ are arbitrary constants. 
Proof. By taking $k=2$ in the statement of Lemma 3.4 we get

$$
\begin{aligned}
{\left[D_{x}, Z_{6}\right]=} & -\left(\alpha_{0} u_{0, x}+2 \alpha_{1} u_{1, x}\right) Z_{6}+\cdots \\
{\left[D_{x}, Z_{7}\right]=} & -\left(\alpha_{2} u_{2, x}+2 \alpha_{1} u_{1, x}\right) Z_{7}-\left(Y_{1}\left(\alpha_{1} u_{1, x}\right)+2 Y_{1}\left(\alpha_{2} u_{2, x}\right)\right) Z_{4}+\cdots \\
{\left[D_{x}, Z_{8}\right]=} & -\left(\alpha_{0} u_{0, x}+2 \alpha_{1} u_{1, x}+\alpha_{2} u_{2, x}\right) Z_{8}+Y_{0}\left(\alpha_{1} u_{1, x}\right) Z_{7}+Y_{2}\left(\alpha_{1} u_{1, x}\right) Z_{6} \\
& -\left(Y_{1}\left(\alpha_{1} u_{1, x}\right)+Y_{1}\left(\alpha_{0} u_{0, x}+\alpha_{2} u_{2, x}\right)\right) Z_{5}+\cdots
\end{aligned}
$$

Now we apply the operator $\operatorname{ad}_{D_{x}}$ to both sides of (4.14) and then simplify due to the relations (4.17), (4.18), (4.19). Comparison of the coefficients before $Z_{7}$ and $Z_{6}$ implies $\lambda=0$ and $\mu=0$. Thus formula (4.14) is simplified

$$
Z_{8}=\nu Z_{5}+\rho Z_{4}+\kappa Z_{3}+\sigma Z_{2}+\delta Z_{1}+\eta Z_{0}
$$

In what follows we will use the following commutativity relations

$$
\begin{aligned}
{\left[D_{x}, Z_{8}\right]=} & -\left(a_{2}+2 a_{1}+a_{0}\right) Z_{8}+Y_{0}\left(a_{1}\right) Z_{7}-Y_{2}\left(a_{1}\right) Z_{6}-Y_{1}\left(a_{2}+a_{1}+a_{0}\right) Z_{5} \\
& +Y_{1} Y_{0}\left(a_{1}\right) Z_{4}-Y_{1} Y_{2}\left(a_{1}\right) Z_{3}+\left(Y_{1} Y_{2} Y_{0}\left(a_{1}\right)+Z_{5}\left(a_{1}\right)\right) Z_{1} \\
{\left[D_{x}, Z_{5}\right]=} & -\left(a_{0}+a_{1}+a_{2}\right) Z_{5}+Y_{0}\left(a_{1}\right) Z_{4}-Y_{2}\left(a_{1}\right) Z_{3}+Y_{2} Y_{0}\left(a_{1}\right) Z_{1} .
\end{aligned}
$$

Let us apply $\operatorname{ad}_{D_{x}}$ to (4.20) then simplify by using (4.21), (4.22), (4.20) and gather the coefficients at $Z_{5}$

$$
-\left(a_{2}+2 a_{1}+a_{0}\right) \nu-Y_{1}\left(a_{2}+a_{1}+a_{0}\right)=D_{x}(\nu)-\left(a_{2}+a_{1}+a_{0}\right) \nu
$$

or the same

$$
D_{x}(\nu)=-a_{1} \nu-Y_{1}\left(a_{2}+a_{1}+a_{0}\right) .
$$

Equation (4.23) implies that $\nu$ depends on three variables $\nu=\nu\left(u, u_{1}, u_{2}\right)$ and splits down into three equations as follows

$$
\begin{aligned}
& \nu_{u}=-\alpha_{0, u_{1}}, \\
& \nu_{u_{1}}=-\alpha_{1} \nu-\alpha_{1, u_{1}}-\alpha_{1}^{2}, \\
& \nu_{u_{2}}=-\alpha_{2, u_{1}} .
\end{aligned}
$$

Substituting $\alpha$ defined by (4.12) into (4.24) and integrating with respect to $u$, we obtain

$$
\nu=-\frac{P^{\prime}\left(u_{1}\right)}{P\left(u_{1}\right)+Q\left(u_{0}\right)}+H\left(u_{1}, u_{2}\right) .
$$

From equation (4.26) we find

$$
\nu=-\frac{Q^{\prime}\left(u_{1}\right)}{P\left(u_{2}\right)+Q\left(u_{1}\right)}+R\left(u_{0}, u_{1}\right) .
$$

Comparison of (4.27) and (4.28) yields

$$
-\frac{P^{\prime}\left(u_{1}\right)}{P\left(u_{1}\right)+Q\left(u_{0}\right)}+H\left(u_{1}, u_{2}\right)=-\frac{Q^{\prime}\left(u_{1}\right)}{P\left(u_{2}\right)+Q\left(u_{1}\right)}+R\left(u, u_{1}\right) .
$$

Due to the fact that variables $u_{0}, u_{1}, u_{2}$ are independent we obtain

$$
-\frac{P^{\prime}\left(u_{1}\right)}{P\left(u_{1}\right)+Q\left(u_{0}\right)}-R\left(u_{0}, u_{1}\right)=-\frac{Q^{\prime}\left(u_{1}\right)}{P\left(u_{2}\right)+Q\left(u_{1}\right)}-H\left(u_{1}, u_{2}\right)=-A\left(u_{1}\right) .
$$


Hence

$$
H\left(u_{1}, u_{2}\right)=-\frac{Q^{\prime}\left(u_{1}\right)}{P\left(u_{2}\right)+Q\left(u_{1}\right)}+A\left(u_{1}\right)
$$

and then

$$
\nu=-\frac{P^{\prime}\left(u_{1}\right)}{P\left(u_{1}\right)+Q\left(u_{0}\right)}-\frac{Q^{\prime}\left(u_{1}\right)}{P\left(u_{2}\right)+Q\left(u_{1}\right)}+A\left(u_{1}\right) .
$$

Note that $\lambda_{(R)}$ defined by (4.11) satisfies the equation (4.7), i.e.,

$$
\lambda_{(R), u}=-\alpha_{0} \lambda_{(R)}-\alpha_{0, u_{0}}-\alpha_{0}^{2} .
$$

Then

$$
\lambda_{(R) 1, u_{1}}=-\alpha_{1} \lambda_{(R) 1}-\alpha_{1, u_{1}}-\alpha_{1}^{2},
$$

where $\lambda_{(R) 1}=D_{n}\left(\lambda_{(R)}\right)$. Here $D_{n}$ is a shift operator $D_{n} u_{k}=u_{k+1}$. Let us subtract (4.30) from $(4.25)$

$$
\left(\nu-\lambda_{(R) 1}\right)_{u_{1}}=-\alpha_{1}\left(\nu-\lambda_{(R) 1}\right) .
$$

Substituting functions (4.11) and (4.29) into the last equation we arrive at the equality

$$
\begin{aligned}
& -\frac{P^{\prime}\left(u_{1}\right) B\left(u_{1}\right)}{P\left(u_{1}\right)+Q\left(u_{0}\right)}-\frac{Q^{\prime}\left(u_{1}\right) B\left(u_{1}\right)}{P\left(u_{2}\right)+Q\left(u_{1}\right)} \\
& \quad+\frac{1}{2}\left(\log Q^{\prime}\left(u_{1}\right) P^{\prime}\left(u_{1}\right)\right)^{\prime}\left(\frac{Q^{\prime}\left(u_{1}\right)}{P\left(u_{2}\right)+Q\left(u_{1}\right)}-\frac{P^{\prime}\left(u_{1}\right)}{P\left(u_{1}\right)+Q\left(u_{0}\right)}+B\left(u_{1}\right)\right) \\
& \quad=\frac{Q^{\prime \prime}\left(u_{1}\right)}{P\left(u_{2}\right)+Q\left(u_{1}\right)}-\frac{P^{\prime \prime}\left(u_{1}\right)}{P\left(u_{1}\right)+Q\left(u_{0}\right)}+B^{\prime}\left(u_{1}\right),
\end{aligned}
$$

where $B\left(u_{1}\right)=A\left(u_{1}\right)-\frac{Q^{\prime \prime}\left(u_{1}\right)}{Q^{\prime}\left(u_{1}\right)}$. This equality is satisfied only if the following conditions hold

$$
\begin{aligned}
& Q^{\prime \prime}\left(u_{1}\right)=-Q^{\prime}\left(u_{1}\right) B\left(u_{1}\right)+\frac{1}{2} Q^{\prime}\left(u_{1}\right)\left(\log Q^{\prime}\left(u_{1}\right) P^{\prime}\left(u_{1}\right)\right)^{\prime}, \\
& P^{\prime \prime}\left(u_{1}\right)=P^{\prime}\left(u_{1}\right) B\left(u_{1}\right)+\frac{1}{2} P^{\prime}\left(u_{1}\right)\left(\log Q^{\prime}\left(u_{1}\right) P^{\prime}\left(u_{1}\right)\right)^{\prime}, \\
& B^{\prime}\left(u_{1}\right)=\frac{1}{2} B\left(u_{1}\right)\left(\log Q^{\prime}\left(u_{1}\right) P^{\prime}\left(u_{1}\right)\right)^{\prime} .
\end{aligned}
$$

The equation (4.33) is satisfied if $B\left(u_{1}\right)=0$ or

$$
\left(\log B\left(u_{1}\right)\right)^{\prime}=\frac{1}{2}\left(\log Q^{\prime}\left(u_{1}\right) P^{\prime}\left(u_{1}\right)\right)^{\prime} .
$$

If $B\left(u_{1}\right)=0$ then $Q\left(u_{1}\right)=c_{1} P\left(u_{1}\right)+c_{2}$ and

$$
\begin{aligned}
& \alpha_{0}=\frac{P^{\prime}\left(u_{0}\right)}{P\left(u_{0}\right)+c_{1} P\left(u_{-1}\right)+c_{2}}+\frac{c_{1} P^{\prime}\left(u_{0}\right)}{P\left(u_{1}\right)+c_{1} P\left(u_{0}\right)+c_{2}}-\frac{P^{\prime \prime}\left(u_{0}\right)}{P^{\prime}\left(u_{0}\right)}, \\
& \lambda_{(M)}:=\nu=-\frac{P^{\prime}\left(u_{1}\right)}{P\left(u_{1}\right)+c_{1} P\left(u_{0}\right)+c_{2}}-\frac{c_{1} P^{\prime}\left(u_{1}\right)}{P\left(u_{2}\right)+c_{1} P\left(u_{1}\right)+c_{2}}+\frac{Q^{\prime \prime}\left(u_{1}\right)}{Q^{\prime}\left(u_{1}\right)}, \\
& \lambda_{(R)}=-\frac{2 c_{1} P^{\prime}\left(u_{0}\right)}{P\left(u_{1}\right)+c_{1} P\left(u_{0}\right)+c_{2}}+\frac{P^{\prime \prime}\left(u_{0}\right)}{P^{\prime}\left(u_{0}\right)} \\
& \lambda_{(L)}=-\frac{2 P^{\prime}\left(u_{1}\right)}{P\left(u_{1}\right)+c_{1} P\left(u_{0}\right)+c_{2}}+\frac{P^{\prime \prime}\left(u_{1}\right)}{P^{\prime}\left(u_{1}\right)} .
\end{aligned}
$$

Here $c_{1} \neq 0$. 
If $B\left(u_{1}\right) \neq 0$ then from the system of equations (4.31), (4.32), and (4.34) we obtain that $Q\left(u_{1}\right)=-\frac{c_{1}}{r\left(u_{1}\right)}+c_{2}, P\left(u_{1}\right)=c_{3} r\left(u_{1}\right)+c_{4}$ and

$$
\begin{aligned}
\alpha_{0}= & \frac{c_{3} r\left(u_{-1}\right) r^{\prime}\left(u_{0}\right)}{c_{3} r\left(u_{0}\right) r\left(u_{-1}\right)+c_{4} r\left(u_{-1}\right)-c_{1}+c_{2} r\left(u_{-1}\right)} \\
& +\frac{c_{1} r^{\prime}\left(u_{0}\right)}{r\left(u_{0}\right)\left(c_{3} r\left(u_{1}\right) r\left(u_{0}\right)+c_{4} r\left(u_{0}\right)-c_{1}+c_{2} r\left(u_{0}\right)\right)}-\frac{r^{\prime \prime}\left(u_{0}\right) r\left(u_{0}\right)-r^{\prime 2}\left(u_{0}\right)}{r\left(u_{0}\right) r^{\prime}\left(u_{0}\right)}, \\
\lambda_{(M)}:= & \nu=-\frac{c_{3} r\left(u_{0}\right) r^{\prime}\left(u_{1}\right)}{c_{3} r\left(u_{1}\right) r\left(u_{0}\right)+c_{4} r\left(u_{0}\right)-c_{1}+c_{2} r\left(u_{0}\right)} \\
& -\frac{c_{1} r^{\prime}\left(u_{1}\right)}{r\left(u_{1}\right)\left(c_{3} r\left(u_{2}\right) r\left(u_{1}\right)+c_{4} r\left(u_{1}\right)-c_{1}+c_{2} r\left(u_{1}\right)\right)} \\
& \quad+\frac{r^{\prime}\left(u_{1}\right)}{r\left(u_{1}\right)}-\frac{2 r^{\prime 2}\left(u_{1}\right)-r^{\prime \prime}\left(u_{1}\right) r\left(u_{1}\right)}{r\left(u_{1}\right) r^{\prime}\left(u_{1}\right)}, \\
\lambda_{(R)}= & -\frac{2 c_{1} r^{\prime}\left(u_{0}\right)}{r\left(u_{0}\right)\left(c_{3} r\left(u_{1}\right) r\left(u_{0}\right)+c_{4} r\left(u_{0}\right)-c_{1}+c_{2} r\left(u_{0}\right)\right)}+\frac{\left.r^{\prime \prime}\right) r\left(u_{0}\right)-2 r^{\prime 2}\left(u_{0}\right)}{r\left(u_{0}\right) r^{\prime}\left(u_{0}\right)}
\end{aligned}
$$

Now let us apply ad $\operatorname{Dox}_{x}$ to (4.20) using (4.21), (4.22), (2.7) and the facts that $Z_{4}=D_{n}\left(Z_{3}\right)$ and $\left[Z_{1}, Z_{4}\right]=D_{n}\left[Z_{0}, Z_{3}\right]=-D_{n}\left(W_{2}\right)=-D_{n}\left(\lambda_{(R)}\right) W_{1}=D_{n}\left(\lambda_{(R)}\right) Z_{4}$ and write down coefficients before $Z_{4}$

$$
-\left(a_{2}+2 a_{1}+a_{0}\right) \rho+Y_{1} Y_{0}\left(a_{1}\right)+Y_{0}\left(a_{1}\right) D_{n}\left(\lambda_{(R)}\right)=\nu Y_{0}\left(a_{1}\right)+D_{x}(\rho)-\left(a_{1}+a_{2}\right) \rho .
$$

Then

$$
D_{x}(\rho)=-\left(a_{1}+a_{0}\right) \rho+Y_{1} Y_{0}\left(a_{1}\right)+Y_{0}\left(a_{1}\right) D_{n}\left(\lambda_{(R)}\right)-\nu Y_{0}\left(a_{1}\right) .
$$

The equation (4.41) implies that $\rho=\rho\left(u, u_{1}, u_{2}\right)$ and splits down into three equations as follows

$$
\rho_{u_{2}}=0, \quad-\alpha_{0} \rho=\rho_{u_{0}}, \quad-\alpha_{1} \rho+\alpha_{1, u_{0} u_{1}}+\alpha_{1} \alpha_{1, u_{0}}+\alpha_{1, u_{0}} D_{n}\left(\lambda_{(R)}\right)-\nu \alpha_{1, u_{0}}=\rho_{u_{1}} .
$$

If $\alpha_{0}, \nu$, and $\lambda_{(R)}$ are defined by the formulas (4.15), (4.35), and (4.36) or by the formulas (4.16), (4.38), and (4.39) correspondingly then $\rho=0$ and the last equations are satisfied.

Now let us apply $\operatorname{ad}_{D_{x}}$ to (4.20) using (4.21), (4.22), (2.7), (4.13) and write down coefficients before $Z_{3}$

$$
-\left(a_{2}+2 a_{1}+a_{0}\right) \kappa-Y_{1} Y_{2}\left(a_{1}\right)-Y_{2}\left(a_{1}\right) \lambda_{(L)}=-\nu Y_{2}\left(a_{1}\right)+D_{x}(\kappa)-\left(a_{1}+a_{2}\right) \kappa .
$$

Then

$$
D_{x}(\kappa)=-\left(a_{2}+a_{1}\right) \kappa-Y_{1} Y_{2}\left(a_{1}\right)-Y_{2}\left(a_{1}\right) \lambda_{(L)}+\nu Y_{2}\left(a_{1}\right) .
$$

The equations (4.42) implies that $\kappa=\kappa\left(u_{1}, u_{2}\right)$ and splits down into two equations as follows

$$
\kappa_{u_{2}}=-\alpha_{2} \kappa, \quad \kappa_{u_{1}}=-\alpha_{1} \kappa-\alpha_{1, u_{2} u_{1}}-\alpha_{1} \alpha_{1, u_{2}}-\alpha_{1, u_{2}} \lambda_{(L)}+\nu \alpha_{1, u_{2}} .
$$

If $\alpha_{0}, \nu$, and $\lambda_{(L)}$ are defined by the formulas (4.15), (4.35), and (4.37) or by the formulas (4.16), (4.38), and (4.40) correspondingly then $\kappa=0$ and the last equations are satisfied. 
Apply $\operatorname{ad}_{D_{x}}$ to (4.20) taking into account that $\rho=\kappa=0$ and write down coefficients before operators $Z_{2}, Z_{1}$ and $Z_{0}$

$$
\begin{aligned}
& D_{x}(\sigma)=-\left(2 a_{1}+a_{0}\right) \sigma, \\
& D_{x}(\delta)=-\left(a_{2}+a_{1}+a_{0}\right) \delta-Y_{1} Y_{2} Y_{0}\left(a_{1}\right)+\lambda Y_{2} Y_{0}\left(a_{1}\right), \\
& D_{x}(\eta)=-\left(a_{2}+2 a_{1}\right) .
\end{aligned}
$$

From these equations we obtain that $\sigma=\delta=\eta=0$.

Thus we have proved that if the expansions (4.1), (4.14) hold then (4.14) should be as follows

$$
Z_{8}=\lambda_{(M)} Z_{5}
$$

Or the same

$$
\left[Y_{1},\left[Y_{2},\left[Y_{1}, Y_{0}\right]\right]\right]=\lambda_{(M)}\left[Y_{2},\left[Y_{1}, Y_{0}\right]\right]
$$

where $\lambda_{M}$ defined by the formula (4.35) or (4.38) and $\alpha_{0}, \lambda_{(R)}$, and $\lambda_{(L)}$ are defined by the formulas (4.15), (4.36), and (4.37) or by the formulas (4.16), (4.39), and (4.40) correspondingly.

Corollary of Theorems 4.1 and 4.2 :

Corollary 4.3. In both cases $Q\left(u_{1}\right)=-\frac{c_{1}}{r\left(u_{1}\right)}+c_{2}, P\left(u_{1}\right)=c_{3} r\left(u_{1}\right)+c_{4}$ and $Q\left(u_{1}\right)=c_{1} P\left(u_{1}\right)+c_{2}$ the constraint (4.3) is satisfied identically.

In a similar way we check that the same conditions (4.15), (4.16) provides the representations

$$
\begin{aligned}
& {\left[Y_{0},\left[Y_{2},\left[Y_{1}, Y_{0}\right]\right] t\right]=\lambda_{(R)}\left[Y_{2},\left[Y_{1}, Y_{0}\right]\right]} \\
& {\left[Y_{2},\left[Y_{2},\left[Y_{1}, Y_{0}\right]\right]\right]=D_{n}\left(\lambda_{(L)}\left[Y_{2},\left[Y_{1}, Y_{0}\right]\right]\right.}
\end{aligned}
$$

\section{Comments on the classification result}

In this section we briefly discuss the statements of Theorems 4.2 and 5.2 (see below) claiming that the lattice (1.1) is integrable in the sense of Definition 1.1 only for two choices of the function $\alpha$ given by (4.15) and (4.16). In both cases the lattice has a functional freedom which is removed by an appropriate point transformation. Therefore we have

Theorem 5.1. Any lattice (1.1) integrable in the sense above is reduced by the point transformation $v=p(u)$ to the following lattice

$$
v_{n, x y}=v_{n, x} v_{n, y}\left(\frac{1}{v_{n}-v_{n-1}}-\frac{1}{v_{n+1}-v_{n}}\right) \text {. }
$$

Specify the point transformations ${ }^{1}$ applied to the lattices. Change of the variables $w=P(u)$ reduces $(4.15)$ to

$$
w_{n, x y}=w_{n, x} w_{n, y}\left(\frac{1}{w_{n}+c_{1} w_{n-1}+c_{2}}+\frac{c_{1}}{w_{n+1}+c_{1} w_{n}+c_{2}}\right) .
$$

The latter is connected with (5.1) by the change of the variables $v_{n}=\left(-c_{1}\right)^{n} w_{n}-\frac{c_{2}}{1+c_{1}}$ if $c_{1} \neq-1$ and by $v_{n}=w_{n}-c_{2} n$ in the special case $c_{1}=-1$.

\footnotetext{
${ }^{1}$ We are glad to acknowledge that these transformations are found by R.I. Yamilov and R.N. Garifullin (private communication).
} 
Change of the variables $v=r(u)$ reduces (4.16) to

$$
v_{n, x y}=v_{n, x} v_{n, y}\left(\frac{v_{n-1}}{v_{n} v_{n-1}+\beta v_{n-1}-\gamma}+\frac{v_{n+1}+\beta}{v_{n} v_{n+1}+\beta v_{n}-\gamma}\right),
$$

where we denote $\beta=\frac{c_{2}+c_{4}}{c_{3}}, \gamma=c_{1} / c_{3}$. Then change of the variables $v=\beta\left(\frac{1}{w}+c\right), \gamma=\beta^{2}\left(c^{2}+c\right)$ reduces $(5.3)$ to

$$
w_{n, x y}=\frac{1}{w_{n}+\frac{c}{c+1} w_{n-1}+\frac{1}{c+1}}+\frac{\frac{c}{c+1}}{w_{n+1}+\frac{c}{c+1} w_{n}+\frac{1}{c+1}} .
$$

The latter coincides with (5.2) if $c_{1}=\frac{c}{c+1}, c_{2}=\frac{1}{c+1}$.

Note that equation (5.1) coincides with the Ferapontov-Shabat-Yamilov equation found in [20] and [4].

Theorem 5.2. The characteristic Lie rings in $x$-and $y$-directions for the following system of hyperbolic type equations

$$
\begin{aligned}
& v_{-1}=c_{0}, \\
& v_{n, x y}=v_{n, x} v_{n, y}\left(\frac{1}{v_{n}-v_{n-1}}-\frac{1}{v_{n+1}-v_{n}}\right), \quad 0 \leq n \leq N, \\
& v_{N+1}=c_{1},
\end{aligned}
$$

are of finite dimension.

The proof of Theorem 5.2 can be found in Appendix A.

Corollary 5.3. The system (5.4) is Darboux integrable.

Remark 5.4. The following lattice (see [20])

$$
\begin{aligned}
& q_{n, x y}=q_{n, x} q_{n, y}\left(f\left(q_{n+1}-q_{n}\right)-f\left(q_{n}-q_{n-1}\right)\right), \\
& f^{\prime}=f^{2}-b^{2}
\end{aligned}
$$

is reduced by the point transformation to (5.1). Namely if $b \neq 0$ then $f(q)=b \tan (b(q+c))=$ $-i b \tanh (i b(q+c))$, where $c$ is the constant of integration, $i$ is the imaginary unit. So we have the lattice

$$
q_{n, x y}=q_{n, x} q_{n, y}(-i b)\left(\tanh \left(i b\left(q_{n+1}-q_{n}+c\right)\right)-\tanh \left(i b\left(q_{n}-q_{n-1}+c\right)\right)\right) .
$$

The change of variables $q_{n}=-\frac{i}{b} v_{n}-n c$ reduces the last lattice to (5.1). If $b=0$ then $f(q)=\frac{1}{q+c}$. By the change of variables $q_{n}=v_{n}-n c$ we obtain (5.1).

\section{Conclusion}

In [9] it was conjectured that any nonlinear integrable two-dimensional lattice of the form

$$
u_{n, x y}=g\left(u_{n+1}, u_{n}, u_{n-1}, u_{n, x}, u_{n, y}\right)
$$

admits cut-off conditions reducing the lattice to a finite system of the hyperbolic type partial differential equations being integrable in the sense of Darboux when they are imposed at two points $n=N_{1}$ and $N_{2}$ chosen arbitrary. 
In the present article we discussed the classification algorithm based on that conjecture. Actually we solved a problem of the complete description of the lattices (1.1) satisfying the suggested requirement. The lattice (1.1) is a particular case of the lattice (6.1) for which the mentioned cut-off condition is easily found: $u_{N_{1}}=c_{0}, u_{N_{2}}=c_{1}$. This circumstance essentially simplifies the situation. Nevertheless even in general when a priori the cut-off condition is also unknown the algorithm might be effective since the assumption on the existence of such boundary conditions puts severe restrictions on the characteristic operators.

We show that the class of integrable lattices of the form (1.1) contains only one model up to the point transformations. This model coincides with the Ferapontov-Shabat-Yamilov equation. The one-dimensional reduction $x=y$ of this lattice satisfies completely also the symmetry integrability conditions (see [23]).

\section{A Appendix}

The goal of the appendix is to prove Theorem 5.2. Let us introduce a special notation $Y_{i_{k}, \ldots, i_{0}}$ for the multiple commutators. It is defined consecutively

$$
Y_{i_{k}, \ldots, i_{0}}=\left[Y_{i_{k}}, Y_{i_{k-1}, \ldots, i_{0}}\right]
$$

Number $k$ is called the order of the operator (A.1).

In order to prove Theorem 5.2 we show that the ring $R(y, N)$ is of finite dimension. Actually we construct the basis in $R(y, N)$ containing the operators

$$
\left\{Y_{i}\right\}_{i=0}^{N}, \quad\left\{Y_{i+1, i}\right\}_{i=0}^{N-1}, \quad\left\{Y_{i+2, i+1, i}\right\}_{i=0}^{N-2}, \quad \ldots, \quad Y_{N, N-1, \ldots, 0} .
$$

\section{A.1 The base case of the mathematical induction}

In the previous section we have proved that

$$
\begin{aligned}
& {\left[Y_{0}, Y_{10}\right]=\lambda_{(R)} Y_{10}, \quad\left[Y_{1}, Y_{10}\right]=\lambda_{(L)} Y_{10},} \\
& {\left[Y_{0}, Y_{210}\right]=\lambda_{(R)} Y_{210}, \quad\left[Y_{1}, Y_{210}\right]=\lambda_{(M)} Y_{210}, \quad\left[Y_{2}, Y_{210}\right]=D_{n}\left(\lambda_{(L)} Y_{210} .\right.}
\end{aligned}
$$

In what follows we will use the following relations which are easily verified

$$
\begin{aligned}
& {\left[D_{x}, Y_{3210}\right]=-\left(a_{3}+a_{2}+a_{1}+a_{0}\right) Y_{3210}-Y_{3}\left(a_{2}\right) Y_{210}+Y_{0}\left(a_{1}\right) Y_{321}, } \\
& {\left[D_{x},\left[Y_{0}, Y_{3210}\right]=-\left(a_{3}+a_{2}+a_{1}+2 a_{0}\right)\left[Y_{0}, Y_{3210}\right]\right.} \\
&-Y_{0}\left(2 a_{1}+a_{0}\right) Y_{3210}-Y_{3}\left(a_{2}\right)\left[Y_{0}, Y_{210}\right]+Y_{0} Y_{0}\left(a_{1}\right) Y_{321}, \\
& {\left[D_{x},\left[Y_{1}, Y_{3210}\right]\right]=}-\left(a_{3}+a_{2}+2 a_{1}+a_{0}\right)\left[Y_{1}, Y_{3210}\right]-Y_{1}\left(a_{2}+a_{1}+a_{0}\right) Y_{3210} \\
&-Y_{1} Y_{3}\left(a_{2}\right) Y_{210}-Y_{3}\left(a_{2}\right)\left[Y_{1}, Y_{210}\right]+Y_{1} Y_{0}\left(a_{1}\right) Y_{321} \\
&+Y_{0}\left(a_{1}\right)\left[Y_{1}, Y_{321}\right] \\
& {\left[D_{x},\left[Y_{2}, Y_{3210}\right]\right]=}-\left(a_{3}+2 a_{2}+a_{1}+a_{0}\right)\left[Y_{2}, Y_{3210}\right]-Y_{2}\left(a_{3}+a_{2}+a_{1}\right) Y_{3210} \\
&-Y_{2} Y_{3}\left(a_{2}\right) Y_{210}-Y_{3}\left(a_{2}\right)\left[Y_{2}, Y_{210}\right]+Y_{2} Y_{0}\left(a_{1}\right) Y_{321} \\
&+Y_{0}\left(a_{1}\right)\left[Y_{2}, Y_{321}\right], \\
& {\left[D_{x},\left[Y_{3}, Y_{3210}\right]\right]=}-\left(2 a_{3}+a_{2}+a_{1}+a_{0}\right)\left[Y_{3}, Y_{3210}\right]-Y_{3}\left(a_{3}+2 a_{2}\right) Y_{3210} \\
&-Y_{3} Y_{3}\left(a_{2}\right) Y_{210}+Y_{0}\left(a_{1}\right)\left[Y_{3}, Y_{321}\right] .
\end{aligned}
$$

We prove the theorem by the mathematical induction. The base case consists in proving a lot of the formulas concerned to small order commutators up to order six. When constructing a linear expression for a given element in $R(y, N)$ as a linear combination of those from (A.2) 
we always use Lemma 2.3. That is why we need in explicit expressions for $\left[D_{x}, Y_{i_{k}, \ldots, i_{0}}\right]$. In the base case we prove a large set of the equalities. Since they all are proved by one and the same way we concentrate on one of them.

Lemma A.1. We have

$$
\left[Y_{0}, Y_{3210}\right]=\lambda_{(R)} Y_{3210}
$$

Proof. By applying the operator $\operatorname{ad}_{D_{x}}$ to $Z=\left[Y_{0}, Y_{3210}\right]-\lambda_{(R)} Y_{3210}$ and simplifying due to the equations (A.5), (A.6) we obtain $\left[D_{x}, Z\right]=0$. Evidently $Z$ satisfies the settings of Lemma 2.3. Due to this lemma we obtain $Z=0$. Lemma A.1 is proved.

In what follows we need in the formulas

$$
\begin{aligned}
& {\left[Y_{1}, Y_{3210}\right]=\lambda_{(M)} Y_{3210}} \\
& {\left[Y_{2}, Y_{3210}\right]=D_{n}\left(\lambda_{(M)}\right) Y_{3210}} \\
& {\left[Y_{3}, Y_{3210}\right]=D_{n}^{2}\left(\lambda_{(L)}\right) Y_{3210}}
\end{aligned}
$$

which are some versions of the formula (A.10) from Lemma A.1.

Now using formulas

$$
\begin{aligned}
{\left[D_{x}, Y_{43210}\right]=-\left(a_{4}\right.} & \left.+a_{3}+a_{2}+a_{1}+a_{0}\right) Y_{43210}-Y_{4}\left(a_{3}\right) Y_{3210}+Y_{0}\left(a_{1}\right) Y_{4321}, \\
{\left[D_{x},\left[Y_{0}, Y_{43210}\right]=\right.} & -\left(a_{4}+a_{3}+a_{2}+a_{1}+2 a_{0}\right)\left[Y_{0}, Y_{43210}\right] \\
& -Y_{0}\left(2 a_{1}+a_{0}\right) Y_{43210}-Y_{4}\left(a_{3}\right)\left[Y_{0}, Y_{3210}\right]+Y_{0} Y_{0}\left(a_{1}\right) Y_{4321}, \\
{\left[D_{x},\left[Y_{1}, Y_{43210}\right]\right]=} & -\left(a_{4}+a_{3}+a_{2}+2 a_{1}+a_{0}\right)\left[Y_{1}, Y_{43210}\right] \\
& -Y_{4}\left(a_{3}\right)\left[Y_{1}, Y_{3210}\right]-Y_{1}\left(a_{2}+a_{1}+a_{0}\right) Y_{43210}-Y_{1} Y_{4}\left(a_{3}\right) Y_{3210} \\
& +Y_{1} Y_{0}\left(a_{1}\right) Y_{4321}+Y_{0}\left(a_{1}\right)\left[Y_{1}, Y_{4321}\right], \\
{\left[D_{x},\left[Y_{2}, Y_{43210}\right]\right]=} & -\left(a_{4}+a_{3}+a_{2}+a_{1}+a_{0}\right)\left[Y_{2}, Y_{43210}\right] \\
& -Y_{2}\left(a_{3}+a_{2}+a_{1}\right) Y_{43210}-Y_{2} Y_{4}\left(a_{3}\right) Y_{3210}-Y_{4}\left(a_{3}\right)\left[Y_{2}, Y_{3210}\right] \\
& +Y_{2} Y_{0}\left(a_{1}\right) Y_{4321}+Y_{0}\left(a_{1}\right)\left[Y_{2}, Y_{4321}\right], \\
{\left[D_{x},\left[Y_{3}, Y_{43210}\right]\right]=} & -\left(a_{4}+2 a_{3}+a_{2}+a_{1}+a_{0}\right)\left[Y_{3}, Y_{43210}\right] \\
& -Y_{3}\left(a_{4}+a_{3}+a_{2}\right) Y_{43210}-Y_{3} Y_{4}\left(a_{3}\right) Y_{3210}-Y_{4}\left(a_{3}\right)\left[Y_{3}, Y_{3210}\right] \\
& +Y_{3} Y_{0}\left(a_{1}\right) Y_{4321}+Y_{0}\left(a_{1}\right)\left[Y_{3}, Y_{4321}\right], \\
{\left[D_{x},\left[Y_{4}, Y_{43210}\right]\right]=} & -\left(2 a_{4}+a_{3}+a_{2}+a_{1}+a_{0}\right)\left[Y_{4}, Y_{43210}\right] \\
& -Y_{4}\left(a_{4}+2 a_{3}\right) Y_{43210}-Y_{4} Y_{4}\left(a_{3}\right) Y_{3210}+Y_{0}\left(a_{1}\right)\left[Y_{4}, Y_{4321}\right]
\end{aligned}
$$

by direct calculations we prove that

$$
\begin{aligned}
& {\left[Y_{0}, Y_{43210}\right]=\lambda_{(R)} Y_{43210},} \\
& {\left[Y_{1}, Y_{43210}\right]=\lambda_{(M)} Y_{43210},} \\
& {\left[Y_{2}, Y_{43210}\right]=D_{n}\left(\lambda_{(M)}\right) Y_{43210},} \\
& {\left[Y_{3}, Y_{43210}\right]=D_{n}^{2}\left(\lambda_{(M)}\right) Y_{43210},} \\
& {\left[Y_{4}, Y_{43210}\right]=D_{n}^{3}\left(\lambda_{(L)}\right) Y_{43210},}
\end{aligned}
$$

where $\lambda_{(R)}, \lambda_{(M)}$, and $\lambda_{(L)}$ are defined by the formulas (4.36), (4.35), and (4.37) or by the formulas (4.39), (4.38), and (4.40) correspondingly.

Now, having explicit formulas for the small order commutators we are ready to work out an induction hypothesis. 


\section{A.2 Inductive step}

Theorem A.2. For $n>1$ the multi-commutators satisfy the following formulas

$$
\begin{aligned}
{\left[D_{x}, Y_{n+1, n, \ldots, 0}\right]=- } & \left(\sum_{i=0}^{n+1} a_{i}\right) Y_{n+1, n, \ldots, 0}-Y_{n+1}\left(a_{n}\right) Y_{n, n-1, \ldots, 0}+Y_{0}\left(a_{1}\right) Y_{n+1, n, \ldots, 1}, \\
{\left[D_{x},\left[Y_{0}, Y_{n+1, n, \ldots, 0}\right]\right]=} & -\left(a_{n+1}+\cdots+a_{1}+2 a_{0}\right)\left[Y_{0}, Y_{n+1, n, \ldots, 0}\right]-Y_{0}\left(a_{0}+2 a_{1}\right) Y_{n+1, n, \ldots, 0} \\
& -Y_{n+1}\left(a_{n}\right)\left[Y_{0}, Y_{n, n-1, \ldots, 0}\right]+Y_{0} Y_{0}\left(a_{1}\right) Y_{n+1, n, \ldots, 1} \\
{\left[D_{x},\left[Y_{k}, Y_{n+1, n, \ldots, 0}\right]\right]=} & -\left(a_{n+1}+\cdots+2 a_{k}+\cdots+a_{0}\right)\left[Y_{k}, Y_{n+1, n, \ldots, 0}\right] \\
- & Y_{k}\left(\sum_{i=0}^{n+1} a_{i}\right) Y_{n+1, n, \ldots, 0}-Y_{k} Y_{n+1}\left(a_{n}\right) Y_{n, n-1, \ldots, 0} \\
- & Y_{n+1}\left(a_{n}\right)\left[Y_{k}, Y_{n, n-1, \ldots, 0}\right]+Y_{k} Y_{0}\left(a_{1}\right) Y_{n+1, n, \ldots, 1} \\
+ & Y_{0}\left(a_{1}\right)\left[Y_{k}, Y_{n+1, n, \ldots, 1}\right], \quad k=1,2, \ldots, n, \\
{\left[D_{x},\left[Y_{n+1}, Y_{n+1, n, \ldots, 0}\right]\right]=} & -\left(2 a_{n+1}+a_{n}+\cdots+a_{0}\right)\left[Y_{n+1}, Y_{n+1, n, \ldots, 0}\right] \\
& -Y_{n+1}\left(a_{n+1}+2 a_{n}\right) Y_{n+1, n, \ldots, 0}-Y_{n+1} Y_{n+1}\left(a_{n}\right) Y_{n, n-1, \ldots, 0} \\
& +Y_{0}\left(a_{1}\right)\left[Y_{n+1}, Y_{n+1, n, \ldots, 1}\right]
\end{aligned}
$$

Proof by induction. For $n=2$ and $n=3$ formulas (A.25)-(A.28) are previously proved (see (A.5)-(A.9) and (A.14)-(A.19)).

Assume that the multi-commutators satisfy the following formulas

$$
\begin{aligned}
{\left[D_{x}, Y_{n, \ldots, 0}\right]=-(} & \left.\sum_{i=0}^{n} a_{i}\right) Y_{n, \ldots, 0}-Y_{n}\left(a_{n-1}\right) Y_{n-1, \ldots, 0}+Y_{0}\left(a_{1}\right) Y_{n, \ldots, 1}, \\
{\left[D_{x},\left[Y_{0}, Y_{n, \ldots, 0}\right]\right]=} & -\left(a_{n}+\cdots+a_{1}+2 a_{0}\right)\left[Y_{0}, Y_{n, \ldots, 0}\right] \\
& -Y_{0}\left(a_{0}+2 a_{1}\right) Y_{n, \ldots, 0}-Y_{n}\left(a_{n-1}\right)\left[Y_{0}, Y_{n-1, \ldots, 0}\right]+Y_{0} Y_{0}\left(a_{1}\right) Y_{n, \ldots, 1}, \\
{\left[D_{x},\left[Y_{k}, Y_{n, \ldots, 0}\right]\right]=} & -\left(a_{n}+\cdots+2 a_{k}+\cdots+a_{0}\right)\left[Y_{k}, Y_{n, \ldots, 0}\right]-Y_{k}\left(\sum_{i=0}^{n} a_{i}\right) Y_{n, \ldots, 0} \\
& -Y_{k} Y_{n}\left(a_{n-1}\right) Y_{n-1, \ldots, 0}-Y_{n}\left(a_{n-1}\right)\left[Y_{k}, Y_{n-1, \ldots, 0}\right]+Y_{k} Y_{0}\left(a_{1}\right) Y_{n, \ldots, 1} \\
& +Y_{0}\left(a_{1}\right)\left[Y_{k}, Y_{n, \ldots, 1}\right], \quad k=1,2, \ldots, n-1, \\
{\left[D_{x},\left[Y_{n}, Y_{n, \ldots, 0}\right]\right]=} & -\left(2 a_{n}+a_{n-1}+\cdots+a_{0}\right)\left[Y_{n}, Y_{n, \ldots, 0}\right] \\
& -Y_{n}\left(a_{n}+2 a_{n-1}\right) Y_{n, \ldots, 0}-Y_{n} Y_{n}\left(a_{n-1}\right) Y_{n-1, \ldots, 0}+Y_{0}\left(a_{1}\right)\left[Y_{n}, Y_{n, \ldots, 1}\right] .
\end{aligned}
$$

Then from these assumptions we deduce similar equations for $n+1$

$$
\begin{aligned}
& {\left[D_{x}, Y_{n+1, n, \ldots, 0}\right]=\left[D_{x},\left[Y_{n+1}, Y_{n, n-1, \ldots, 0}\right]\right] } \\
&=\left[Y_{n+1},\left[D_{x}, Y_{n, n-1, \ldots, 0}\right]-\left[Y_{n, n-1, \ldots, 0},\left[D_{x}, Y_{n+1}\right]\right]\right. \\
&= {\left[Y_{n+1},-\left(a_{n}+a_{n-1}+\cdots+a_{0}\right) Y_{n, n-1, \ldots, 0}-Y_{n}\left(a_{n-1}\right) Y_{n-1, n-2, \ldots, 0}\right.} \\
&\left.+Y_{0}\left(a_{1}\right) Y_{n, n-1, \ldots, 1}\right]-\left[Y_{n, n-1, \ldots, 0},-a_{n+1} Y_{n+1}\right] \\
&=-\left(\sum_{i=0}^{n+1} a_{i}\right) Y_{n+1, n, \ldots, 0}-Y_{n+1}\left(a_{n}+a_{n-1}+\cdots+a_{0}\right) Y_{n, n-1, \ldots, 0} Y_{n, n-1, \ldots, 0} \\
&-Y_{n+1} Y_{n}\left(a_{n-1}\right) Y_{n-1, n-2, \ldots, 0}-Y_{n}\left(a_{n-1}\right)\left[Y_{n+1}, Y_{n-1, n-2, \ldots, 0}\right] \\
&+Y_{n+1} Y_{0}\left(a_{1}\right) Y_{n, n-1, \ldots, 1}+Y_{0}\left(a_{1}\right) Y_{n+1, n, \ldots, 1}+Y_{n, n-1, \ldots, 0}\left(a_{n+1}\right) Y_{n+1} .
\end{aligned}
$$

Note that

$$
Y_{i}\left(a_{j}\right)=0 \quad \text { if } \quad|i-j|>1,
$$


i.e., if $i \neq j, i \neq j \pm 1$, and

$$
\left[Y_{m}, Y_{n, \ldots, 0}\right]=0 \quad \text { if } \quad m-n>1
$$

That is why the following terms in (A.29) are equal to zero

$$
\begin{aligned}
& Y_{n+1}\left(a_{n-1}+\cdots+a_{0}\right)=0, \quad Y_{n+1} Y_{n}\left(a_{n-1}\right)=0, \\
& {\left[Y_{n+1}, Y_{n-1, n-2, \ldots, 0}\right]=0, \quad Y_{n+1} Y_{0}\left(a_{1}\right)=0, \quad Y_{n, n-1, \ldots, 0}\left(a_{n+1}\right)=0 .}
\end{aligned}
$$

Thus the equality (A.29) takes the form (A.25).

Let us prove the formula (A.26),

$$
\begin{aligned}
{\left[D_{x},\left[Y_{0}, Y_{n+1, n, \ldots, 0}\right]\right]=} & {\left[Y_{0},\left[D_{x}, Y_{n+1, n, \ldots, 0}\right]\right]-\left[Y_{n+1, n, \ldots, 0},\left[D_{x}, Y_{0}\right]\right] } \\
= & {\left[Y_{0},-\left(\sum_{i=0}^{n+1} a_{i}\right) Y_{n+1, n, \ldots, 0}-Y_{n+1}\left(a_{n}\right) Y_{n, n-1, \ldots, 0}+Y_{0}\left(a_{1}\right) Y_{n+1, n, \ldots, 1}\right] } \\
& -\left[Y_{n+1, n, \ldots, 0},-a_{0} Y_{0}\right] .
\end{aligned}
$$

From this equality using property of linearity of the commutators and the equations (A.30) we obtain the formula (A.26). The formulas (A.27) and (A.28) are proved in a similar way.

Theorem A.3. For $m \geq 1$ the multi-commutators satisfy the following formulas

$$
\begin{aligned}
& {\left[Y_{0}, Y_{m+1, m, \ldots, 0}\right]=\lambda_{(R)} Y_{m+1, m, \ldots, 0},} \\
& {\left[Y_{k}, Y_{m+1, m, \ldots, 0}\right]=D_{n}^{k-1}\left(\lambda_{(M)}\right) Y_{m+1, m, \ldots, 0}, \quad k=1, \ldots, m,} \\
& {\left[Y_{m+1}, Y_{m+1, m, \ldots, 0}\right]=D_{n}^{m}\left(\lambda_{(L)}\right) Y_{m+1, m, \ldots, 0} .}
\end{aligned}
$$

Proof by induction. For $m=1,2,3$ formulas (A.31)-(A.33) are true (see (A.3), (A.4), (4.43), (A.10), (A.11), (A.12), (A.13), (A.20)-(A.24)).

Assume that the multi-commutators satisfy the following formulas

$$
\begin{aligned}
& {\left[Y_{0}, Y_{m, m-1, \ldots, 0}\right]=\lambda_{(R)} Y_{m, m-1, \ldots, 0},} \\
& {\left[Y_{k}, Y_{m, m-1, \ldots, 0}=D_{n}^{k-1}\left(\lambda_{(M)}\right) Y_{m, m-1, \ldots, 0}, \quad k=1, \ldots, m-1,\right.} \\
& {\left[Y_{m}, Y_{m, m-1, \ldots, 0}\right]=D_{n}^{m-1}\left(\lambda_{(L)}\right) Y_{m, m-1, \ldots, 0} .}
\end{aligned}
$$

Let us first prove the formula (A.31). The proof is rather tricky: we assume the expansion with undetermined coefficients

$$
\begin{aligned}
{\left[Y_{0}, Y_{m+1, m, \ldots, 0}\right]=} & \lambda Y_{m+1, m, \ldots, 0}+\mu Y_{m+1, m, \ldots, 1}+\nu Y_{m, m-1, \ldots, 0}+\epsilon Y_{m+1, m, \ldots, 2} \\
& +\eta Y_{m, m-1, \ldots, 1}+\zeta Y_{m-1, m-2, \ldots, 0}+\cdots \\
& +\theta Y_{m+1, m}+\cdots+\xi Y_{10}+\sigma Y_{m+1}+\cdots+\delta Y_{0}
\end{aligned}
$$

and then evaluate the coefficients consecutively in the following way. We apply the operator $\operatorname{ad}_{D_{x}}$ to (A.37) and gather the coefficients before the linearly independent operators. For instance, by comparing the coefficients before the multi-commutator $Y_{m+1, m, \ldots, 0}$ and then using formulas from Theorem 2.1 with $n=m-1$ we find

$$
D_{x}(\lambda)=-a_{0} \lambda-Y_{0}\left(a_{0}+2 a_{1}\right) .
$$

The latter coincides with the equation (4.4) and, therefore, we can conclude that $\lambda=\lambda_{(R)}$. 
Compare now the coefficients before $Y_{m+1, m, \ldots, 1}$ to get an equation for determining $\mu$. Note that by Theorem A.2 we have

$$
\begin{aligned}
& {\left[D_{x}, Y_{m+1, m, \ldots, 1}\right]=D_{n}\left[D_{x}, Y_{m, m-1, \ldots, 0}\right]=\mid \text { Theorem A.2 }} \\
& \quad=D_{n}\left(-\left(\sum_{i=0}^{m} a_{i}\right) Y_{m, m-1, \ldots, 0}-Y_{m}\left(a_{m-1}\right) Y_{m-1, m-2, \ldots, 0}+Y_{0}\left(a_{1}\right) Y_{m, m-1, \ldots, 1}\right) \\
& \quad=-\left(\sum_{i=1}^{m+1} a_{i}\right) Y_{m+1, m, \ldots, 1}-Y_{m+1}\left(a_{m}\right) Y_{m, m-1, \ldots, 1}+Y_{1}\left(a_{2}\right) Y_{m+1, m, \ldots, 2} .
\end{aligned}
$$

Due to the relation (A.38) the desired equation reduces to the form

$$
D_{x}(\mu)=-2 a_{0} \mu+Y_{0} Y_{0}\left(a_{1}\right)-\lambda Y_{0}\left(a_{1}\right) .
$$

It is easily checked that the equation has the only solution $\mu=0$. Continuing this way we can prove that all of the other coefficients $\nu, \epsilon, \ldots, \delta$ in (A.37) vanish. Now for the operator $Z=\left[Y_{0}, Y_{m+1, m, \ldots, 0}\right]-\lambda_{(R)} Y_{m+1, m, \ldots, 0}$ we have $\left[D_{x}, Z\right]=0$. Due to Lemma 2.3 it implies $Z=0$. That completes the proof of the formula (A.31).

Now we prove the formula (A.32). To this end we assume that the equation holds

$$
\begin{aligned}
{\left[Y_{k}, Y_{m+1, m, \ldots, 0}\right]=} & \lambda Y_{m+1, m, \ldots, 0}+\mu Y_{m+1, m, \ldots, 1}+\nu Y_{m, m-1, \ldots, 0}+\epsilon Y_{m+1, m, \ldots, 2} \\
& +\eta Y_{m, m-1, \ldots, 1}+\zeta Y_{m-1, m-2, \ldots, 0}+\cdots \\
& +\theta Y_{m+1, m}+\cdots+\xi Y_{10}+\sigma Y_{m+1}+\cdots+\delta Y_{0}
\end{aligned}
$$

with the coefficients to be determined.

Let us apply $\operatorname{ad}_{D_{x}}$ to (A.39) and write down the coefficients before the operator $Y_{m+1, m, \ldots, 0}$

$$
D_{x}(\lambda)=-a_{k} \lambda-Y_{k}\left(\sum_{i=0}^{m+1} a_{i}\right) \text {. }
$$

Apply $D_{n}^{-(k-1)}$ to the last equation

$$
D_{x}\left(D_{n}^{-(k-1)}(\lambda)\right)=-a_{1} D_{n}^{-(k-1)}(\lambda)-Y_{1}\left(a_{2}+a_{1}+a_{0}\right) .
$$

This equation coincides with the equation (4.23) then $D_{n}^{-(k-1)}(\lambda)=\lambda_{(M)}$ and

$$
\lambda=D_{n}^{k-1}\left(\lambda_{(M)}\right) .
$$

Note that due to the formula (A.35) we have

$$
\begin{aligned}
{\left[Y_{k}, Y_{m+1, m, \ldots, 1}\right] } & =D_{n}\left[Y_{k-1}, Y_{m, m-1, \ldots, 0}\right] \\
& =D_{n}\left(D_{n}^{k-2}\left(\lambda_{(M)}\right) Y_{m, m-1, \ldots, 0}\right)=D_{n}^{k-1}\left(\lambda_{(M)}\right) Y_{m+1, m, \ldots, 1}
\end{aligned}
$$

Apply $\operatorname{ad}_{D_{x}}$ to (A.39) using the formulas from Theorem A.2 and the formula (A.40) and write down the coefficients before the multi-commutator $Y_{m+1, m, \ldots, 1}$

$$
\begin{gathered}
-\left(a_{m+1}+\cdots+2 a_{k}+\cdots+a_{0}\right) \mu+Y_{k} Y_{0}\left(a_{1}\right)+Y_{0}\left(a_{1}\right) D_{n}^{k-1}\left(\lambda_{(M)}\right. \\
=\lambda Y_{0}\left(a_{1}\right)+D_{x}(\mu)-\left(\sum_{i=1}^{m+1}\right) \mu .
\end{gathered}
$$


Since $\lambda=D_{n}^{k-1}\left(\lambda_{(M)}\right)$ the latter can be brought to the form

$$
D_{x}(\mu)=-\left(a_{0}+a_{k}\right) \mu+Y_{k} Y_{0}\left(a_{1}\right) .
$$

Evaluate the action of the product of the operators

$$
Y_{k} Y_{0}\left(a_{1}\right)=\left(\frac{\partial}{\partial u_{k}}+\alpha_{k} u_{k, x} \frac{\partial}{\partial u_{k, x}}\right)\left(\alpha_{1, u} u_{1, x}\right)= \begin{cases}\alpha_{1, u u_{1}} u_{1, x}+\alpha_{1} \alpha_{1, u} u_{1, x}, & k=1 \\ \alpha_{1, u u_{2}} u_{1, x}, & k=2 \\ 0, & k>2\end{cases}
$$

Thus if $k=1$ then the equality (A.41) takes the form

$$
D_{x}(\mu)=-\left(\alpha_{0} u_{x}+\alpha_{1} u_{1, x}\right) \mu+\left(\alpha_{1, u u_{1}}+\alpha_{1} \alpha_{1, u}\right) u_{1, x} .
$$

This equation implies that $\mu=\mu\left(u, u_{1}\right)$ and splits down into two equations as follows

$$
\mu_{u}=-\alpha_{0} \mu, \quad \mu_{u_{1}}=-\alpha_{1} \mu+\alpha_{1, u u_{1}}+\alpha_{1} \alpha_{1, u} .
$$

Then we can prove that $\mu=0$.

If $k=2$ then the equality (A.41) takes the form

$$
D_{x}(\mu)=-\left(\alpha_{0} u_{x}+\alpha_{2} u_{2, x}\right) \mu+\alpha_{1, u u_{2}} u_{1, x} .
$$

This equation implies that $\mu=\mu\left(u, u_{1}, u_{2}\right)$ and splits down into three equations as follows

$$
\mu_{u}=-\alpha_{0} \mu, \quad \mu_{u_{1}}=\alpha_{1, u u_{2}}, \quad \mu_{u_{2}}=-\alpha_{2} \mu .
$$

And then again $\mu=0$.

If $k>2$ then the equality (A.41) takes the form

$$
D_{x}(\mu)=-\left(\alpha_{0} u_{x}+\alpha_{k} u_{k, x}\right) \mu .
$$

This equation implies that $\mu=\mu\left(u, u_{k}\right)$ and splits down into two equations as follows

$$
\mu_{u}=-\alpha_{0} \mu, \quad \mu_{u_{k}}=-\alpha_{k} \mu .
$$

Then $\mu=0$.

In a similar way we can verify that all of the coefficients in (A.39) vanish except $\lambda$. Thus due to Lemma 2.3 formula (A.32) is correct.

Now we check the formula (A.33). First we assume that the following decomposition takes place

$$
\begin{aligned}
{\left[Y_{m+1}, Y_{m+1, m, \ldots, 0}\right]=} & \lambda Y_{m+1, m, \ldots, 0}+\mu Y_{m+1, m, \ldots, 1}+\nu Y_{m, m-1, \ldots, 0}+\epsilon Y_{m+1, m, \ldots, 2} \\
& +\eta Y_{m, m-1, \ldots, 1}+\zeta Y_{m-1, m-2, \ldots, 0}+\cdots+\theta Y_{m+1, m}+\cdots \\
& +\xi Y_{10}+\sigma Y_{m+1}+\cdots+\delta Y_{0}
\end{aligned}
$$

with undefined factors.

Let us apply $\operatorname{ad}_{D_{x}}$ to (A.42) and write down the coefficients before the multi-commutator $Y_{m+1, m, \ldots, 0}$

$$
D_{x}(\lambda)=-a_{m+1} \lambda-Y_{m+1}\left(a_{m+1}+2 a_{m}\right) .
$$

Apply $D_{n}^{-m}$ to this equation

$$
D_{x}\left(D_{n}^{-m}(\lambda)\right)=-a_{1} D_{n}^{-m}(\lambda)-Y_{1}\left(a_{1}+2 a_{0}\right) .
$$

This equation coincides with (4.4) then $D_{n}^{-m}(\lambda)=\lambda_{(L)}$ and $\lambda=D_{n}^{m}\left(\lambda_{(L)}\right)$. 
Let us apply $\operatorname{ad}_{D_{x}}$ to (A.42) and write down the coefficients before the multi-commutator $Y_{m+1, m, \ldots, 1}$

$$
-\left(2 a_{m+1}+a_{m}+\cdots+a_{0}\right) \mu+Y_{0}\left(a_{1}\right) D_{n}^{m}\left(\lambda_{(L)}\right)=\lambda Y_{0}\left(a_{1}\right)+D_{x}(\mu)-\left(\sum_{i=1}^{m+1} a_{i}\right) \mu .
$$

Note that $\lambda=D_{n}^{m}\left(\lambda_{(L)}\right)$ then the last equality takes the form

$$
D_{x}(\mu)=-\left(a_{m+1}+a_{0}\right) \mu .
$$

Then $\mu=0$.

Let us apply $\operatorname{ad}_{D_{x}}$ to (A.42) and write down the coefficients before the multi-commutator $Y_{m, m-1, \ldots, 0}$

$$
D_{x}(\nu)=-2 a_{m+1} \nu-Y_{m+1} Y_{m+1}\left(a_{m}\right)+\lambda Y_{m+1}\left(a_{m}\right) .
$$

Note that

$$
\begin{aligned}
& \lambda Y_{m+1}\left(a_{m}\right)-Y_{m+1} Y_{m+1}\left(a_{m}\right) \\
& \left.\quad=D_{n}^{m}\left(\lambda_{(L)}\right) Y_{m+1}\left(a_{m}\right)-Y_{m+1} Y_{m+1}\left(a_{m}\right)=D_{m}\left(\lambda_{(L)}\right) Y_{1}\left(a_{0}\right)-Y_{1} Y_{1}\left(a_{0}\right)\right)=0 .
\end{aligned}
$$

Then the equation (A.43) takes the form $D_{x}(\nu)=-2 a_{m+1} \nu$ and we obtain that $\nu=0$.

In a similar way we can prove the vanishing of the other coefficients in (A.42). Now by applying Lemma 2.3 it is easy to complete the proof of the formula (A.33). Theorem A.3 is proved.

\section{Acknowledgments}

The authors are grateful to the anonymous referees for their critical remarks and fruitful recommendations.

\section{References}

[1] Adler V.E., Habibullin I.T., Boundary conditions for integrable chains, Funct. Anal. Appl. 31 (1997), 75-85.

[2] Adler V.E., Shabat A.B., Yamilov R.I., Symmetry approach to the integrability problem, Theoret. and Math. Phys. 125 (2000), 1603-1661.

[3] Bogdanov L.V., Konopelchenko B.G., Grassmannians $\operatorname{Gr}(N-1, N+1)$, closed differential $N-1$-forms and N-dimensional integrable systems, J. Phys. A: Math. Theor. 46 (2013), 085201, 17 pages, arXiv:1208.6129.

[4] Ferapontov E.V., Laplace transforms of hydrodynamic-type systems in Riemann invariants, Theoret. and Math. Phys. 110 (1997), 68-77, solv-int/9705017.

[5] Ferapontov E.V., Khusnutdinova K.R., On the integrability of $(2+1)$-dimensional quasilinear systems, Comm. Math. Phys. 248 (2004), 187-206, nlin.SI/0305044.

[6] Ferapontov E.V., Khusnutdinova K.R., Tsarev S.P., On a class of three-dimensional integrable Lagrangians, Comm. Math. Phys. 261 (2006), 225-243, nlin.SI/0407035.

[7] Gubbiotti G., Scimiterna C., Yamilov R.I., Darboux integrability of trapezoidal $H^{4}$ and $H^{6}$ families of lattice equations II: general solutions, arXiv:1704.05805.

[8] Gürel B., Habibullin I.T., Boundary conditions for two-dimensional integrable chains, Phys. Lett. A 233 (1997), 68-72.

[9] Habibullin I.T., Characteristic Lie rings, finitely-generated modules and integrability conditions for $(2+1)-$ dimensional lattices, Phys. Scripta 87 (2013), 065005, 5 pages, arXiv:1208.5302.

[10] Habibullin I.T., Pekcan A., Characteristic Lie algebra and classification of semidiscrete models, Theoret. and Math. Phys. 151 (2007), 781-790, nlin.SI/0610074. 
[11] Levi D., Winternitz P., Lie point symmetries and commuting flows for equations on lattices, J. Phys. A: Math. Gen. 35 (2002), 2249-2262, math-ph/0112007.

[12] Mañas M., Martínez Alonso L., Álvarez-Fernández C., The multicomponent 2D Toda hierarchy: discrete flows and string equations, Inverse Problems 25 (2009), 065007, 31 pages, arXiv:0809.2720.

[13] Mikhailov A.V., Shabat A.B., Sokolov V.V., The symmetry approach to classification of integrable equations, in What is Integrability?, Springer Ser. Nonlinear Dynam., Springer, Berlin, 1991, 115-184.

[14] Mikhailov A.V., Yamilov R.I., Towards classification of (2+1)-dimensional integrable equations. Integrability conditions. I, J. Phys. A: Math. Gen. 31 (1998), 6707-6715.

[15] Moser J., Finitely many mass points on the line under the influence of an exponential potential-an integrable system, in Dynamical Systems, Theory and Applications (Rencontres, Battelle Res. Inst., Seattle, Wash., 1974), Lecture Notes in Phys., Vol. 38, Springer, Berlin, 1975, 467-497.

[16] Odesskii A.V., Sokolov V.V., Integrable $(2+1)$-dimensional systems of hydrodynamic type, Theoret. and Math. Phys. 163 (2010), 549-586, arXiv:1009.2778.

[17] Pavlov M.V., Popowicz Z., On integrability of a special class of two-component (2+1)-dimensional hydrodynamic-type systems, SIGMA 5 (2009), 011, 10 pages, arXiv:0901.4312.

[18] Pogrebkov A.K., Commutator identities on associative algebras and the integrability of nonlinear evolution equations, Theoret. and Math. Phys. 154 (2008), 405-417, nlin.SI/0703018.

[19] Shabat A.B., Higher symmetries of two-dimensional lattices, Phys. Lett. A 200 (1995), 121-133.

[20] Shabat A.B., Yamilov R.I., To a transformation theory of two-dimensional integrable systems, Phys. Lett. A 227 (1997), 15-23.

[21] Smirnov S.V., Semidiscrete Toda lattices, Theoret. and Math. Phys. 172 (2012), 1217-1231, arXiv:1203.1764.

[22] Smirnov S.V., Darboux integrability of discrete two-dimensional Toda lattices, Theoret. and Math. Phys. 182 (2015), 189-210, arXiv:1410.0319.

[23] Yamilov R., Symmetries as integrability criteria for differential difference equations, J. Phys. A: Math. Gen. 39 (2006), R541-R623.

[24] Zakharov V.E., Manakov S.V., Construction of higher-dimensional nonlinear integrable systems and of their solutions, Funct. Anal. Appl. 19 (1985), 89-101.

[25] Zheltukhin K., Zheltukhina N., Semi-discrete hyperbolic equations admitting five dimensional characteristic x-ring, J. Nonlinear Math. Phys. 23 (2016), 351-367, arXiv:1604.00221.

[26] Zheltukhin K., Zheltukhina N., Bilen E., On a class of Darboux-integrable semidiscrete equations, Adv. Difference Equ. (2017), 182, 14 pages.

[27] Zhiber A.V., Murtazina R.D., Habibullin I.T., Shabat A.B., Characteristic Lie rings and integrable models in mathematical physics, Ufa Math. J. 4 (2012), 89-101.

[28] Zhiber A.V., Murtazina R.D., Habibullin I.T., Shabat A.B., Characteristic Lie rings and nonlinear integrable equations, Institute of Computer Science, Moscow - Izhevsk, 2012.

[29] Zhiber A.V., Sokolov V.V., Exactly integrable hyperbolic equations of Liouville type, Russ. Math. Surv. 56 (2001), no. 1, 61-101. 